EUROPEAN LABORATORY FOR PARTICLE PHYSICS

CERN-PPE/97-69

June 17, 1997

\title{
Three-prong $\tau$ decays with charged kaons
}

\author{
The ALEPH Collaboration ${ }^{1}$
}

\begin{abstract}
Final states with charged kaons in three-prong $\tau$ decays are studied by exploiting the particle identification from the $\mathrm{dE} / \mathrm{dx}$ measurement. The results are based on a sample of about $1.6 \times 10^{5}$ detected $\tau$ pairs collected with the ALEPH detector between 1991 and 1995 around the $\mathrm{Z}$ peak. The following branching ratios have been measured: $B\left(\tau^{-} \rightarrow K^{-} K^{+} \pi^{-} \nu_{\tau}\right)=(1.63 \pm 0.21 \pm 0.17) \times 10^{-3}$, $B\left(\tau^{-} \rightarrow K^{-} \pi^{+} \pi^{-} \nu_{\tau}\right)=(2.14 \pm 0.37 \pm 0.29) \times 10^{-3}, B\left(\tau^{-} \rightarrow K^{-} K^{+} \pi^{-} \pi^{0} \nu_{\tau}\right)=$ $(0.75 \pm 0.29 \pm 0.15) \times 10^{-3}$, and $B\left(\tau^{-} \rightarrow K^{-} \pi^{+} \pi^{-} \pi^{0} \nu_{\tau}\right)=(0.61 \pm 0.39 \pm 0.18) \times 10^{-3}$. The first two measurements are more precise than the current world averages, while the last two channels are investigated for the first time. The 95\% C.L. upper limit on the branching ratio for the decay $\tau^{-} \rightarrow K^{-} K^{+} K^{-} \nu_{\tau}$ is $0.19 \times 10^{-3}$. A study of intermediate states occurring in the $K^{-} K^{+} \pi^{-} \nu_{\tau}$ and $K^{-} \pi^{+} \pi^{-} \nu_{\tau}$ decays is also presented.
\end{abstract}

(To be submitted to Zeitschrift für Physik C.)

\footnotetext{
${ }^{1}$ See next pages for the list of authors.
} 


\section{The ALEPH Collaboration}

R. Barate, D. Buskulic, D. Decamp, P. Ghez, C. Goy, J.-P. Lees, A. Lucotte, M.-N. Minard, J.-Y. Nief, B. Pietrzyk

Laboratoire de Physique des Particules (LAPP), IN² $P^{3}$-CNRS, 74019 Annecy-le-Vieux Cedex, France

M.P. Casado, M. Chmeissani, P. Comas, J.M. Crespo, M. Delfino, E. Fernandez, M. Fernandez-Bosman, Ll. Garrido, ${ }^{15}$ A. Juste, M. Martinez, G. Merino, R. Miquel, Ll.M. Mir, C. Padilla, I.C. Park, A. Pascual, J.A. Perlas, I. Riu, F. Sanchez, F. Teubert

Institut de Física d'Altes Energies, Universitat Autònoma de Barcelona, 08193 Bellaterra (Barcelona), Spain $^{7}$

A. Colaleo, D. Creanza, M. de Palma, G. Gelao, G. Iaselli, G. Maggi, M. Maggi, N. Marinelli, S. Nuzzo, A. Ranieri, G. Raso, F. Ruggieri, G. Selvaggi, L. Silvestris, P. Tempesta, A. Tricomi, ${ }^{3}$ G. Zito

Dipartimento di Fisica, INFN Sezione di Bari, 70126 Bari, Italy

X. Huang, J. Lin, Q. Ouyang, T. Wang, Y. Xie, R. Xu, S. Xue, J. Zhang, L. Zhang, W. Zhao

Institute of High-Energy Physics, Academia Sinica, Beijing, The People's Republic of China ${ }^{8}$

D. Abbaneo, R. Alemany, U. Becker, A.O. Bazarko, ${ }^{20}$ P. Bright-Thomas, M. Cattaneo, F. Cerutti, G. Dissertori, H. Drevermann, R.W. Forty, M. Frank, R. Hagelberg, J.B. Hansen, J. Harvey, P. Janot, B. Jost, E. Kneringer, J. Knobloch, I. Lehraus, G. Lutters, P. Mato, A. Minten, L. Moneta, A. Pacheco, J.F. Pusztaszeri, ${ }^{23}$ F. Ranjard, G. Rizzo, L. Rolandi, D. Rousseau, D. Schlatter, M. Schmitt, O. Schneider, W. Tejessy, I.R. Tomalin, H. Wachsmuth, A. Wagner ${ }^{24}$

European Laboratory for Particle Physics (CERN), 1211 Geneva 23, Switzerland

Z. Ajaltouni, A. Barrès, C. Boyer, A. Falvard, C. Ferdi, P. Gay, C . Guicheney, P. Henrard, J. Jousset, B. Michel, S. Monteil, J-C. Montret, D. Pallin, P. Perret, F. Podlyski, J. Proriol, P. Rosnet, J.M. Rossignol

Laboratoire de Physique Corpusculaire, Université Blaise Pascal, $I N^{2} P^{3}-C N R S$, Clermont-Ferrand, 63177 Aubière, France

T. Fearnley, J.D. Hansen, J.R. Hansen, P.H. Hansen, B.S. Nilsson, B. Rensch, A. Wäänänen

Niels Bohr Institute, 2100 Copenhagen, Denmark ${ }^{9}$

G. Daskalakis, A. Kyriakis, C. Markou, E. Simopoulou, I. Siotis, A. Vayaki

Nuclear Research Center Demokritos (NRCD), Athens, Greece

A. Blondel, G. Bonneaud, J.C. Brient, P. Bourdon, A. Rougé, M. Rumpf, A. Valassi ${ }^{6}$ M. Verderi, H. Videau

Laboratoire de Physique Nucléaire et des Hautes Energies, Ecole Polytechnique, $I N^{2} P^{3}$-CNRS, 91128

Palaiseau Cedex, France

D.J. Candlin, M.I. Parsons

Department of Physics, University of Edinburgh, Edinburgh EH9 3JZ, United Kingdom ${ }^{10}$

E. Focardi, G. Parrini, K. Zachariadou

Dipartimento di Fisica, Università di Firenze, INFN Sezione di Firenze, 50125 Firenze, Italy

M. Corden, C. Georgiopoulos, D.E. Jaffe

Supercomputer Computations Research Institute, Florida State University, Tallahassee, FL 323064052, USA ${ }^{13,14}$

A. Antonelli, G. Bencivenni, G. Bologna, ${ }^{4}$ F. Bossi, P. Campana, G. Capon, D. Casper, V. Chiarella, G. Felici, P. Laurelli, G. Mannocchi, ${ }^{5}$ F. Murtas, G.P. Murtas, L. Passalacqua, M. Pepe-Altarelli 
L. Curtis, S.J. Dorris, A.W. Halley, I.G. Knowles, J.G. Lynch, V. O'Shea, C. Raine, J.M. Scarr, K. Smith, P. Teixeira-Dias, A.S. Thompson, E. Thomson, F. Thomson, R.M. Turnbull

Department of Physics and Astronomy, University of Glasgow, Glasgow G12 8QQ,United Kingdom ${ }^{10}$

O. Buchmüller, S. Dhamotharan, C. Geweniger, G. Graefe, P. Hanke, G. Hansper, V. Hepp, E.E. Kluge, A. Putzer, J. Sommer, K. Tittel, S. Werner, M. Wunsch

Institut für Hochenergiephysik, Universität Heidelberg, 69120 Heidelberg, Fed. Rep. of Germany ${ }^{16}$

R. Beuselinck, D.M. Binnie, W. Cameron, P.J. Dornan, M. Girone, S. Goodsir, E.B. Martin, A. Moutoussi, J. Nash, J.K. Sedgbeer, P. Spagnolo, A.M. Stacey, M.D. Williams

Department of Physics, Imperial College, London SW7 2BZ, United Kingdom ${ }^{10}$

V.M. Ghete, P. Girtler, D. Kuhn, G. Rudolph

Institut für Experimentalphysik, Universität Innsbruck, 6020 Innsbruck, Austria ${ }^{18}$

A.P. Betteridge, C.K. Bowdery, P. Colrain, G. Crawford, A.J. Finch, F. Foster, G. Hughes, R.W. Jones, T. Sloan, M.I. Williams

Department of Physics, University of Lancaster, Lancaster LA1 4YB, United Kingdom ${ }^{10}$

A. Galla, I. Giehl, A.M. Greene, C. Hoffmann, K. Jakobs, K. Kleinknecht, G. Quast, B. Renk, E. Rohne, H.-G. Sander, P. van Gemmeren, C. Zeitnitz

Institut für Physik, Universität Mainz, 55099 Mainz, Fed. Rep. of Germany ${ }^{16}$

J.J. Aubert, C. Benchouk, A. Bonissent, G. Bujosa, J. Carr, P. Coyle, C. Diaconu, F. Etienne, N. Konstantinidis, O. Leroy, F. Motsch, P. Payre, M. Talby, A. Sadouki, M. Thulasidas, K. Trabelsi

Centre de Physique des Particules, Faculté des Sciences de Luminy, IN ${ }^{2} P^{3}$-CNRS, 13288 Marseille, France

M. Aleppo, M. Antonelli, F. Ragusa ${ }^{2}$

Dipartimento di Fisica, Università di Milano e INFN Sezione di Milano, 20133 Milano, Italy

R. Berlich, W. Blum, V. Büscher, H. Dietl, G. Ganis, C. Gotzhein, H. Kroha, G. Lütjens, G. Lutz, W. Männer, H.-G. Moser, R. Richter, A. Rosado-Schlosser, S. Schael, R. Settles, H. Seywerd, R. St. Denis, H. Stenzel, W. Wiedenmann, G. Wolf

Max-Planck-Institut für Physik, Werner-Heisenberg-Institut, 80805 München, Fed. Rep. of Germany ${ }^{16}$

J. Boucrot, O. Callot, ${ }^{2}$ S. Chen, Y. Choi,${ }^{21}$ A. Cordier, M. Davier, L. Duflot, J.-F. Grivaz, Ph. Heusse, A. Höcker, A. Jacholkowska, M. Jacquet, D.W. Kim, ${ }^{12}$ F. Le Diberder, J. Lefrançois, A.-M. Lutz, I. Nikolic, M.-H. Schune, S. Simion, E. Tournefier, J.-J. Veillet, I. Videau, D. Zerwas

Laboratoire de l'Accélérateur Linéaire, Université de Paris-Sud, $I N^{2} P^{3}-C N R S, 91405$ Orsay Cedex, France

P. Azzurri, G. Bagliesi, G. Batignani, S. Bettarini, C. Bozzi, G. Calderini, M. Carpinelli, M.A. Ciocci, V. Ciulli, R. Dell'Orso, R. Fantechi, I. Ferrante, L. Foà, ${ }^{1}$ F. Forti, A. Giassi, M.A. Giorgi, A. Gregorio, F. Ligabue, A. Lusiani, P.S. Marrocchesi, A. Messineo, F. Palla, G. Sanguinetti, A. Sciabà, J. Steinberger, R. Tenchini, G. Tonelli, ${ }^{19}$ C. Vannini, A. Venturi, P.G. Verdini

Dipartimento di Fisica dell'Università, INFN Sezione di Pisa, e Scuola Normale Superiore, 56010 Pisa, Italy

G.A. Blair, L.M. Bryant, J.T. Chambers, Y. Gao, M.G. Green, T. Medcalf, P. Perrodo, J.A. Strong, J.H. von Wimmersperg-Toeller

Department of Physics, Royal Holloway \& Bedford New College, University of London, Surrey TW20 OEX, United Kingdom ${ }^{10}$

D.R. Botterill, R.W. Clifft, T.R. Edgecock, S. Haywood, P. Maley, P.R. Norton, J.C. Thompson, A.E. Wright

Particle Physics Dept., Rutherford Appleton Laboratory, Chilton, Didcot, Oxon OX11 OQX, United Kingdom $^{10}$ 
B. Bloch-Devaux, P. Colas, S. Emery, W. Kozanecki, E. Lançon, M.C. Lemaire, E. Locci, P. Perez, J. Rander, J.-F. Renardy, A. Roussarie, J.-P. Schuller, J. Schwindling, A. Trabelsi, B. Vallage

CEA, DAPNIA/Service de Physique des Particules, CE-Saclay, 91191 Gif-sur-Yvette Cedex, France ${ }^{17}$

S.N. Black, J.H. Dann, R.P. Johnson, H.Y. Kim, A.M. Litke, M.A. McNeil, G. Taylor

Institute for Particle Physics, University of California at Santa Cruz, Santa Cruz, CA 95064, USA ${ }^{22}$

C.N. Booth, R. Boswell, C.A.J. Brew, S. Cartwright, F. Combley, M.S. Kelly, M. Lehto, W.M. Newton, J. Reeve, L.F. Thompson

Department of Physics, University of Sheffield, Sheffield S3 7RH, United Kingdom ${ }^{10}$

A. Böhrer, S. Brandt, G. Cowan, C. Grupen, P. Saraiva, L. Smolik, F. Stephan

Fachbereich Physik, Universität Siegen, 57068 Siegen, Fed. Rep. of Germany ${ }^{16}$

M. Apollonio, L. Bosisio, R. Della Marina, G. Giannini, B. Gobbo, G. Musolino

Dipartimento di Fisica, Università di Trieste e INFN Sezione di Trieste, 34127 Trieste, Italy

J. Rothberg, S. Wasserbaech

Experimental Elementary Particle Physics, University of Washington, WA 98195 Seattle, U.S.A.

S.R. Armstrong, E. Charles, P. Elmer, D.P.S. Ferguson, S. González, T.C. Greening, O.J. Hayes, H. Hu, S. Jin, P.A. McNamara III, J.M. Nachtman, J. Nielsen, W. Orejudos, Y.B. Pan, Y. Saadi, I.J. Scott, J. Walsh, Sau Lan Wu, X. Wu, J.M. Yamartino, G. Zobernig

Department of Physics, University of Wisconsin, Madison, WI 53706, USA ${ }^{11}$

${ }^{1}$ Now at CERN, 1211 Geneva 23, Switzerland.

${ }^{2}$ Also at CERN, 1211 Geneva 23, Switzerland.

${ }^{3}$ Also at Dipartimento di Fisica, INFN, Sezione di Catania, Catania, Italy.

${ }^{4}$ Also Istituto di Fisica Generale, Università di Torino, Torino, Italy.

${ }^{5}$ Also Istituto di Cosmo-Geofisica del C.N.R., Torino, Italy.

${ }^{6}$ Supported by the Commission of the European Communities, contract ERBCHBICT941234.

${ }^{7}$ Supported by CICYT, Spain.

${ }^{8}$ Supported by the National Science Foundation of China.

${ }^{9}$ Supported by the Danish Natural Science Research Council.

${ }^{10}$ Supported by the UK Particle Physics and Astronomy Research Council.

${ }^{11}$ Supported by the US Department of Energy, grant DE-FG0295-ER40896.

${ }^{12}$ Permanent address: Kangnung National University, Kangnung, Korea.

${ }^{13}$ Supported by the US Department of Energy, contract DE-FG05-92ER40742.

${ }^{14}$ Supported by the US Department of Energy, contract DE-FC05-85ER250000.

${ }^{15}$ Permanent address: Universitat de Barcelona, 08208 Barcelona, Spain.

${ }^{16}$ Supported by the Bundesministerium für Bildung, Wissenschaft, Forschung und Technologie, Fed.

Rep. of Germany.

${ }^{17}$ Supported by the Direction des Sciences de la Matière, C.E.A.

${ }^{18}$ Supported by Fonds zur Förderung der wissenschaftlichen Forschung, Austria.

${ }^{19}$ Also at Istituto di Matematica e Fisica, Università di Sassari, Sassari, Italy.

${ }^{20}$ Now at Princeton University, Princeton, NJ 08544, U.S.A.

${ }^{21}$ Permanent address: Sung Kyun Kwan University, Suwon, Korea.

${ }^{22}$ Supported by the US Department of Energy, grant DE-FG03-92ER40689.

${ }^{23}$ Now at School of Operations Research and Industrial Engineering, Cornell University, Ithaca, NY 14853-3801, U.S.A.

${ }^{24}$ Now at Schweizerischer Bankverein, Basel, Switzerland. 


\section{Introduction}

The study of $\tau$ decays into strange particles is important in order to test low-energy QCD phenomenology [1], SU(3)-symmetry breaking [2] and meson theories [3, 4, 5, 6]. Experimentally, such studies are well advanced for one-prong $\tau$ decays while the situation is still quite poor for the three-prong final states. This is due in part to the relatively small branching ratios and the difficulty in identifying charged particles in a three-track environment, especially at the LEP energies where the produced $\tau$ leptons are strongly Lorentz-boosted.

This paper describes an investigation of three-prong $\tau$ decays involving charged kaons with the measurement of the branching ratios for the decays $\tau^{-} \rightarrow K^{-} \pi^{+} \pi^{-}\left(\pi^{0}\right) \nu_{\tau}$ and $\tau^{-} \rightarrow K^{-} K^{+} \pi^{-}\left(\pi^{0}\right) \nu_{\tau}$ as the main goal (throughout this paper charge-conjugate states are implied). For convenience a same-sign (opposite-sign) charged particle is defined to have the same (opposite) charge as the parent $\tau$. Since the $W^{-}$boson only couples to $\bar{u} d$ or $\bar{u} s$ pairs in $\tau^{-}$decay, the weak current produces states with strangeness $S=0,-1$ only, and consequently the $K^{+} \pi^{-} \pi^{-}\left(\pi^{0}\right)$ and $K^{-} K^{-} \pi^{+}\left(\pi^{0}\right)$ hadronic systems should not be produced. It is therefore possible to identify $K^{-} K^{+} K^{-}$and $K^{-} K^{+} \pi^{-}\left(\pi^{0}\right)$ final states by tagging the opposite-sign kaons, while the decay modes with $K^{-} \pi^{+} \pi^{-}\left(\pi^{0}\right)$ can be selected with their characteristic same-sign kaons after subtracting the $K^{-} K^{+} K^{-}$and the $K^{-} K^{+} \pi^{-}\left(\pi^{0}\right)$ contributions. This correspondence is exploited by fitting $\mathrm{dE} / \mathrm{dx}$ spectra separately for same- and opposite-sign tracks, thus obtaining the relevant branching fractions without having to identify all three particles in the final state. This procedure is based on a precise recalibration of the track $\mathrm{dE} / \mathrm{dx}$ in the environment of the closely bunched three-prong jet of $\tau$ decays.

A more complete particle identification is applied in order to study the dynamics of these decays, particularly the resonance structure. Cuts are applied on the $\mathrm{dE} / \mathrm{dx}$ of all tracks, enhancing the purity of the $K^{-} K^{+} \pi^{-}$and the $K^{-} \pi^{+} \pi^{-}$samples at a cost in efficiency. Of special interest is the production of possible intermediate states such as $K^{*} \pi$ and $K \rho$ proceeding through the $K^{-} \pi^{+} \pi^{-}$decay, since their relative ratio can distinguish the two axial strange resonances $K_{1}(1270)$ and $K_{1}(1400)$. This ratio can be used to estimate the $\mathrm{SU}(3)$-symmetry breaking for the $I=1 / 2$ members of the two octets [2]. Since the $K_{1}(1270)$ is expected to also decay into $K \omega$, it is interesting to study the $K^{-} \pi^{+} \pi^{-} \pi^{0}$ channel, which provides another handle on axial resonance production.

The results presented here are based on the data collected by ALEPH from 1991 to 1995 , corresponding to 201,753 produced $\tau$ pairs.

\section{The ALEPH detector}

A detailed description of the ALEPH detector can be found elsewhere [7]. Only the most important features relevant for this analysis are briefly mentioned in the following.

Charged particle momenta are measured by a magnetic spectrometer consisting of a precision vertex detector, a cylindrical drift chamber (the inner tracking chamber or ITC), and a large time projection chamber (TPC). A $1.5 \mathrm{~T}$ axial magnetic field is provided by a superconducting solenoidal coil. The transverse momentum resolution for high- 
momentum tracks is $\sigma_{p_{t}} / p_{t}=6 \times 10^{-4} p_{t}$, with $p_{t}$ in $\mathrm{GeV} / c$. Photons are measured with an energy resolution $\sigma_{E} / E=0.18 / \sqrt{E}$, with $E$ in $\mathrm{GeV}$, in the electromagnetic calorimeter (ECAL), a 22-radiation-length sandwich of lead plates and proportional wire chambers. The fine readout segmentation in solid-angle $\left(0.9^{\circ} \times 0.9^{\circ}\right)$ is essential for high-energy $\pi^{0}$ reconstruction and the three-fold longitudinal segmentation allows a check of the electromagnetic nature of the detected showers. The $1.2 \mathrm{~m}$ thick iron return yoke of the magnet is interleaved with 23 layers of streamer tubes and acts as a hadronic calorimeter (HCAL).

In the data sample used for this analysis, all components of the detector were required to be operational. Furthermore, one of the following trigger conditions was required: a minimum ECAL energy of $6 \mathrm{GeV}$, or a track segment in the ITC pointing to an energy deposit in ECAL of at least $1.2 \mathrm{GeV}$, or a track segment in the ITC matching the signal of a penetrating particle in HCAL. By comparing redundant and independent triggers involving the tracking detectors and the main calorimeters, the trigger efficiency for $\tau$ pair events is estimated to be better than $99.99 \%$ within the selection criteria used in this analysis.

Of prime interest for this analysis is the measurement of the ionization deposited by charged particles traversing the TPC gas (at atmospheric pressure) which is sampled up to 338 times on the sense wires of the TPC endplates. To avoid the large fluctuations caused by $\delta$-rays, a truncated mean is used, leaving aside the largest $40 \%$ of values. Because threeprong $\tau$ decays suffer from track overlap compared to one-prong $\tau$ decays, the average $K / \pi$ separation decreases from $2.5 \sigma$ for the one-prong to $1.9 \sigma$ for the three-prong $\tau$ decays.

\section{Three-prong $\tau$ event selection}

The standard ALEPH $\tau^{+} \tau^{-}$selection described in Ref [8] is applied. Each event is divided into two hemispheres by means of a plane perpendicular to the thrust axis. The three-prong $\tau$ events are required to have exactly three good charged hadrons, each of which must have at least four TPC coordinates, an impact parameter $d_{0}$ in the plane perpendicular to the beam direction smaller than $2 \mathrm{~cm}$, and the distance from the interaction region along the beam axis within $\pm 10 \mathrm{~cm}$. The contamination from $e^{+} e^{-}$ pairs is estimated by Monte Carlo to be less than $10^{-4}$ and is therefore neglected.

In order to separate the final states with or without an additional $\pi^{0}$, a standard photon and $\pi^{0}$ recognition algorithm is applied [8]. Photon candidates are recognized as conversions or identified by studying their shower development in ECAL. A $\pi^{0}$ candidate is reconstructed by either pairing two photons to form a resolved $\pi^{0}$, or by means of an energy-weighted moment technique (referred to as unresolved $\pi^{0}$ 's). The remaining single photons are assumed to originate from the asymmetric $\pi^{0}$ decay or the high-energy $\pi^{0}$ 's producing a single cluster in ECAL.

To remove the background of hadronic $\mathrm{Z}$ decays, the opposite hemisphere to the selected one is required to be a $\tau$-like hemisphere with only one charged track or an invariant mass smaller than $0.8 \mathrm{GeV} / \mathrm{c}^{2}$. The product of the number of objects (charged tracks and neutral showers) in each hemisphere has to be smaller than 40 and the sum of 
the maximum angle between two tracks in each hemisphere is required to be smaller than $0.25 \mathrm{rad}$. Using these selection criteria, samples with $24,6693 h^{-} \nu_{\tau}$ and $7,9463 h^{-} \pi^{0} \nu_{\tau}$ decays are selected with non- $\tau q \bar{q}$ background contributions estimated to be $(0.27 \pm 0.09) \%$ and $(0.59 \pm 0.20) \%$, respectively.

\section{$4 \mathrm{dE} / \mathrm{dx}$ recalibration for three-prong $\tau$ decays}

The general ALEPH dE/dx calibration for hadronic events is described elsewhere [7]: a parametrization of the Bethe-Bloch formula is fitted to data providing the expected value of the energy loss for a given particle type. However, at LEP, charged tracks in threeprong $\tau$ decays are usually very close to each other. The $\mathrm{dE} / \mathrm{dx}$ reconstruction requires a separation between two tracks of at least $3 \mathrm{~cm}$ in the $z$ direction (where $z$ is defined along the $e^{ \pm}$beams), in order to use the corresponding hits. As a result, the number of samples $\left(N_{S}\right)$ for $\mathrm{dE} / \mathrm{dx}$ measurement is much reduced when compared to the situation prevailing with isolated tracks as found in one-prong decays. Since the expected means of the $\mathrm{dE} / \mathrm{dx}$ for different particles are determined from fits to $\mathrm{dE} / \mathrm{dx}$ in hadronic and leptonic events, including both isolated and overlapping tracks, the $\mathrm{dE} / \mathrm{dx}$ value and the corresponding resolution are not precise enough for the situation of the very collimated jet of tracks found in three-prong $\tau$ decays. For this reason, the $\mathrm{dE} / \mathrm{dx}$ recalibration performed for one-prong $\tau$ decays [9] is not suitable either. A different calibration procedure is therefore designed for this analysis.

Tracks used for the calibration must have a good $\mathrm{dE} / \mathrm{dx}$ measurement with a minimum of 40 initial samples and with no large $\mathrm{dE} / \mathrm{dx}$ deposit (less than twice minimum-ionizing). There are $(76.0 \pm 0.5) \%$ of data and $(75.8 \pm 0.3) \%$ of Monte Carlo tracks in the threeprong $\tau$ decays satisfying this requirement. The track overlap has a dominant effect on the $N_{S}$ distribution. Instead of finding the ideal 338 maximum samples for each charged track, the average $N_{S}$ is reduced to around 200. The situation of track overlap in threeprong $\tau$ events is illustrated in Fig. 1; it is clear that the track overlap is the overwhelming feature controlling $\mathrm{dE} / \mathrm{dx}$ behaviour and calibration in three-prong $\tau$ decay. The extent of track overlap is expected to depend on the angle between any two charged hadrons as shown in Fig. 2. The number of samples is found to be sensitive to the angle in the $r-z$ plane only, which is expected since ionization electrons drift along the $z$ axis onto the wires of the TPC detectors. A good agreement between data and Monte Carlo is observed in both plots, indicating that the simulation of the two-track overlap is satisfactory.

In the process of the $\mathrm{dE} / \mathrm{dx}$ recalibration, the small kaon content is ignored in the first-order step, designed to correct the most significant detector effects. The relevant variable $x_{\pi}$ is defined as

$$
x_{\pi}=\frac{R-R_{\pi}}{\sigma_{\pi}},
$$

where $R, R_{\pi}$ and $\sigma_{\pi}$ are the measured $\mathrm{dE} / \mathrm{dx}$, the expected value for pions, and the corresponding standard deviation. Offsets in the $x_{\pi}$ distribution are governed by the following factors: (i) the period of data acquisition; (ii) the polar angle of the track; (iii) the number of samples; and (iv) the track curvature. The expected mean $\mathrm{dE} / \mathrm{dx}$ of the

pion and its resolution are corrected accordingly. These corrections are applied for each of 


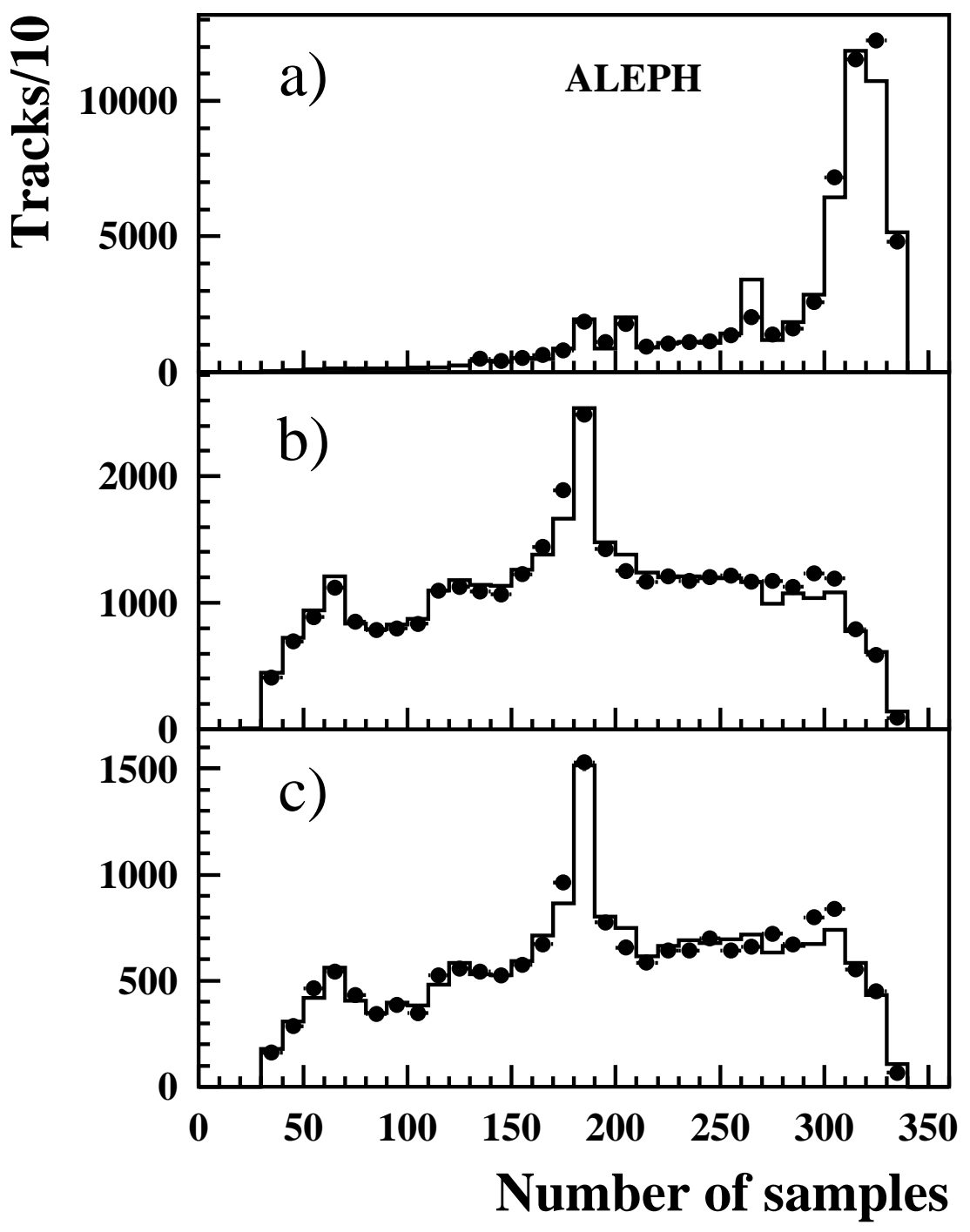

Figure 1: The distributions of the number of $d E / d x$ samples in the TPC for data (dots) and Monte Carlo (histograms). a) one-prong decays, b) same-sign particles in three-prong decays, and c) opposite-sign particles in three-prong decays. The peak observed near to $N_{S} \sim 185$ is related to the geometry of the TPC sectors. 


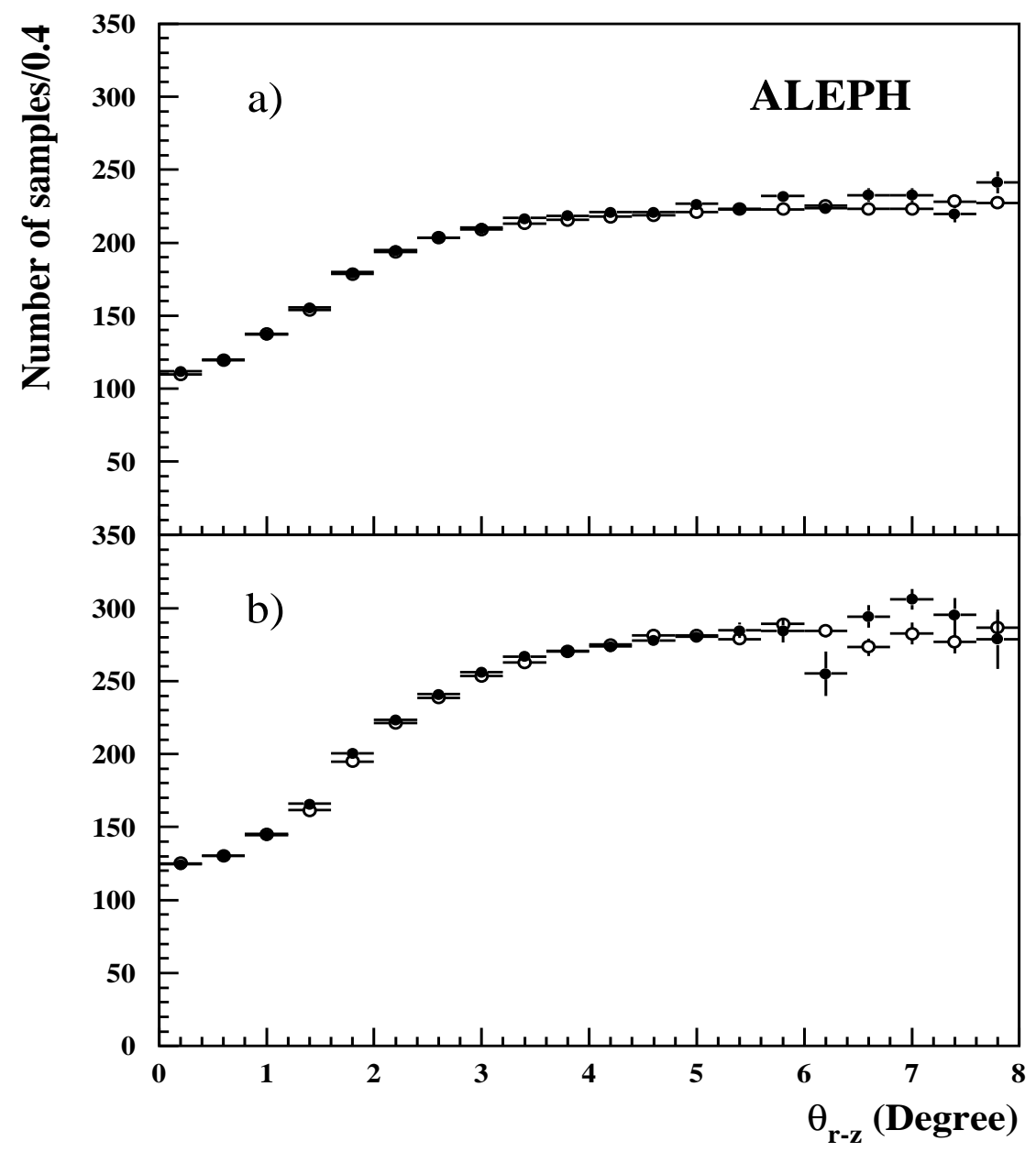

Figure 2: The average number of $d E / d x$ samples in the TPC as a function of the minimum angle between two charged hadrons in the $r$-z plane for data (dots with error bars) and for Monte Carlo (open circles). a) same-sign tracks and b) opposite-sign tracks are studied separately. For a same-sign track the angle is defined with respect to the other same-sign track, while for an opposite-sign track it is taken as the smallest angle with respect to each of the two same-sign tracks. 
the two modes $3 h^{-}$and $3 h^{-} \pi^{0}$, with the same- and opposite-sign tracks treated separately, since the latter suffer less from overlap. A simple Gaussian is fitted to the resulting $x_{\pi}$ distribution, thereby yielding calibration parameters for $R_{\pi}$ and $\sigma_{\pi}$, so that the average of $x_{\pi}$ and its standard deviation are equal to 0 and 1 , respectively.

A second-order absolute recalibration for pions is performed using samples in the $3 h^{-} \nu_{\tau}$ mode with a kaon-reduced content. Either of the two mass combinations should satisfy $M_{\pi^{+} \pi^{-}} \geq 0.85 \mathrm{GeV} / c^{2}$ for same-sign tracks and $M_{\pi^{+} \pi^{-}} \geq 0.8 \mathrm{GeV} / c^{2}$ for opposite-sign tracks: these cuts are designed to reduce the number of kaons from both the $K^{-} \pi^{+} \pi^{-} \nu_{\tau}$ and the $K^{-} K^{+} \pi^{-} \nu_{\tau}$ decay modes. As a result, the relative kaon fraction is reduced by a factor of about 2 for the same-sign tracks and 3 for the opposite-sign tracks. With this kaon-reduced samples, Gaussian fits are again performed. The small remaining kaon contribution can still somewhat bias the calibration parameters, and it will be corrected for in the final pion calibration (Section 6).

For the $3 h^{-} \pi^{0} \nu_{\tau}$ mode, a direct pion sample can be selected by tagging the charged tracks from the $\omega$ decay in $\tau^{-} \rightarrow \omega h^{-} \nu_{\tau}$, satisfying $\left|M_{\gamma \gamma}-M_{\pi^{0}}\right| \leq 80 \mathrm{MeV} / c^{2}$ and $\left|M_{\pi^{+} \pi^{-} \pi^{0}}-M_{\omega}\right| \leq 40 \mathrm{MeV} / c^{2}$. With this sample, the second-order recalibration for the $3 h^{-} \pi^{0} \nu_{\tau}$ mode is performed without the influence of kaon contamination present in the above first-order recalibration.

Finally, the pion resolution function $F_{\pi}\left(x_{\pi}\right)$ in three-prong $\tau$ decays is directly obtained from the $\omega$ sample. This function is slightly asymmetric and is parametrized by a sum of two Gaussians (Fig. 3), fixing the parameters for the first Gaussian at the expected values of 0 and 1 and leaving the parameters for the second Gaussian free to fit a small tail in the high side of $x_{\pi}$. This first-order parametrization of the pion $\mathrm{dE} / \mathrm{dx}$ resolution is limited by the statistics of the $\omega$ sample. A better estimate will be performed in Section 6 , using the full statistics of the three-prong sample while fitting the kaon component. The final and most precise calibration of $\bar{x}_{\pi}(\pi)$ and $\sigma_{\pi}(\pi)$ will then be obtained.

\section{$5 \quad$ Modelling the kaon signal}

The $\tau$ Monte Carlo samples are generated by the standard KORALZ generator [10], where the form factors for three-prong $\tau$ decays are derived from Ref $[4]^{2}$. Some significant discrepancies in the resonance structure are observed between the model predictions and the data as described in the following sections. The efficiencies for $K \pi \pi$ and $K \bar{K} \pi$ modes are computed after making corrections to the model. The $\tau$ decay channels $K^{-} K^{+} \pi^{-} \pi^{0} \nu_{\tau}$, $K^{-} \pi^{+} \pi^{-} \pi^{0} \nu_{\tau}$ and $K^{-} K^{+} K^{-} \nu_{\tau}$ have not been considered before and are not included in the decay library of KORALZ. To remedy this defect, a phase-space generator is used to describe the first two channels. For the $K^{-} K^{+} K^{-} \nu_{\tau}$ channel, a $K \phi$ final state is assumed, which is conservative with respect to the efficiency since only an upper limit will be set. The relevant efficiencies for signal and feedthrough background channels with a correction described in Section 8.1 are given in Table 1. In addition, a sample of 5.8 million Monte Carlo hadronic Z decay is generated with JETSET7.4 [11] to be used for the background

\footnotetext{
${ }^{2}$ It has been pointed out in Ref [5] that the expression for the vector part in the $K^{-} K^{+} \pi^{-} \nu_{\tau}$ channel of the Decker et al. model [4] is incorrect because of a violation of $G$ parity. This has been corrected in the present analysis.
} 


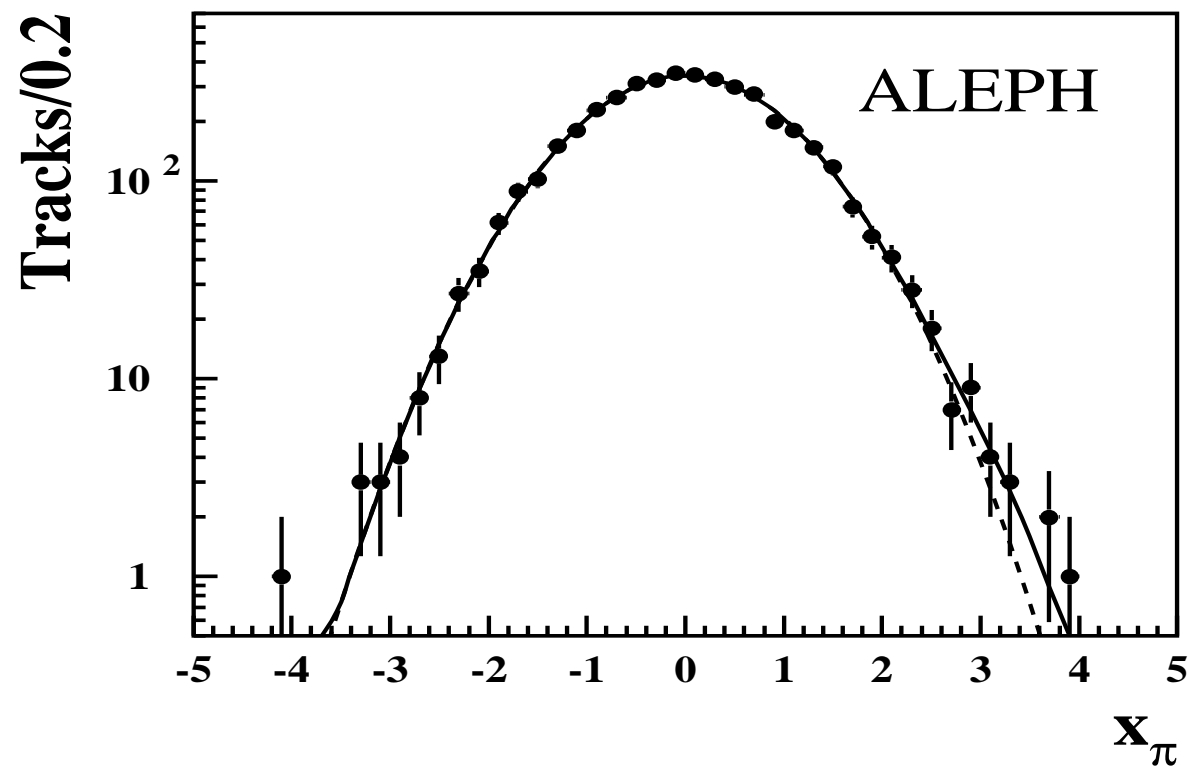

Figure 3: Distribution of $d E / d x$ for pions from $\tau^{-} \rightarrow \omega h^{-} \nu_{\tau}, \omega \rightarrow \pi^{+} \pi^{-} \pi^{0}$. The solid curve is a sum of two Gaussians and the dashed curve represents the single standard Gaussian. See text for details.

estimation.

\section{Fitting the kaon fraction}

\subsection{The $x_{\pi}$ distribution for kaons}

The expected shape of the kaon contribution in the $x_{\pi}$ distribution is derived from the pion resolution function $F_{\pi}\left(x_{\pi}\right)$ given in Section 4 assuming

$$
F_{K}\left(x_{K}\right)=F_{\pi}\left(x_{\pi}\right) .
$$

Values for the reduced variable $x_{K}$ defined analogously to (1) are generated according to the distribution (2). The resulting distribution expected in the $x_{\pi}$ variable reads

$$
G_{K}\left(x_{\pi}\right)=\frac{\sigma_{\pi}^{c}}{\sigma_{K}^{c}} F_{K}\left(\frac{x_{\pi} \sigma_{\pi}^{c}+R_{\pi}^{c}-R_{K}^{c}}{\sigma_{K}^{c}}\right),
$$

where $R_{\pi, K}^{c}$ and $\sigma_{\pi, K}^{c}$ are the track parameters assuming a pion or kaon type and corrected as described in Section 4 using the calibration variables, in particular, the momentum and the number of $\mathrm{dE} / \mathrm{dx}$ samples.

Because the fit of the $x_{\pi}$ distribution is performed by integrating over all final state variables, Monte Carlo modelling of the decay modes with kaons plays an important role in deriving the final kaon distribution. Another factor is the simulation of the $\mathrm{dE} / \mathrm{dx}$ 


\begin{tabular}{|l|c|c|c|c|}
\hline \hline Mode & \multicolumn{2}{|c|}{$3 h^{-} \nu_{\tau}$} & \multicolumn{2}{c|}{$3 h^{-} \pi^{0} \nu_{\tau}$} \\
\hline Efficiency & $\epsilon\left(K^{-}\right)(\%)$ & $\epsilon\left(K^{+}\right)(\%)$ & $\epsilon\left(K^{-}\right)(\%)$ & $\epsilon\left(K^{+}\right)(\%)$ \\
\hline$K^{-} \pi^{+} \pi^{-}$ & $38.20 \pm 0.68$ & - & $0.87 \pm 0.09$ & - \\
\hline$K^{-} K^{+} \pi^{-}$ & $28.83 \pm 0.69$ & $32.73 \pm 0.74$ & $0.87 \pm 0.14$ & $0.97 \pm 0.16$ \\
\hline$K^{-} \pi^{+} \pi^{-} \pi^{0}$ & $3.02 \pm 0.39$ & - & $19.50 \pm 0.49$ & - \\
\hline$K^{-} K^{+} \pi^{-} \pi^{0}$ & $2.31 \pm 0.24$ & $3.22 \pm 0.29$ & $9.20 \pm 0.30$ & $11.53 \pm 0.33$ \\
\hline$K^{-} K^{0}$ & $7.74 \pm 0.35$ & - & $0.16 \pm 0.05$ & - \\
\hline$K^{-} K^{0} \pi^{0}$ & $0.71 \pm 0.12$ & - & $3.30 \pm 0.25$ & - \\
\hline \hline
\end{tabular}

Table 1: Single particle(same-sign and opposite-sign) efficiencies in each channel involved in this analysis. Errors are statistical only.

measurement as far as the track overlap is concerned. Both aspects need to be studied for the systematic effects and to obtain the corresponding uncertainties (Section 8).

The $x_{\pi}$ distribution of hadrons in data is fitted with the following expression

$$
\frac{1}{N} \frac{d N}{d x_{\pi}}=\left(1-f_{K}\right) F_{\pi}\left(x_{\pi} ; \bar{x}_{\pi}, \sigma_{\pi}\right)+f_{K} G_{K}\left(x_{\pi} ; \bar{x}_{\pi}, \sigma_{\pi}\right)
$$

where $N$ is the total number of tracks, and the final calibration parameters in the pion resolution function (2) are used.

\subsection{Final pion calibration and $x_{\pi}$ shape determination}

The technique of statistical particle identification requires a precise description for the $x_{\pi}$ distribution of pions, since the kaon fraction is expected to be small. The final three-prong sample is used to get the most accurate pion resolution function with its final calibration. Because the $\omega$ sample has been used, there is no bias in the second-order recalibration for the $3 h^{-} \pi^{0}$ samples. However, due to the difference of kaon contamination in the second-order $\mathrm{dE} / \mathrm{dx}$ recalibration, the $3 h^{-}$same-sign and the $3 h^{-}$opposite-sign samples are first treated separately in order to correct the biases. A three-parameter fit of the $x_{\pi}$ distribution using $F_{\pi}\left(x_{\pi}\right)$ from the $\omega$ sample is performed to extract the kaon fraction and the $x_{\pi}(\pi)$ parameters for the calibration. The relevant kaon distribution is derived as discussed in Section 6.1. The fit results for $\bar{x}_{\pi}(\pi)$ and $\sigma_{\pi}(\pi)$ are: $0.004 \pm 0.007$ and $1.001 \pm 0.005$ for the same-sign tracks; and $0.000 \pm 0.009$ and $0.998 \pm 0.007$ for the opposite-sign tracks. These pion calibration corrections are applied to the corresponding $3 h^{-}$samples.

It is now justifiable to combine all data together including same- and opposite-sign tracks, and $3 h^{-}$and $3 h^{-} \pi^{0}$ final states, since the $\mathrm{dE} / \mathrm{dx}$ recalibration applied following the procedure described in Section 4 takes into account the dependence on momentum and number of samples. While the distributions of these variables are final-state dependent, the actual corrections parametrized as functions of $p$ and $N_{S}$ are not.

In Fig. 4 the $x_{\pi}$ distribution is shown for full statistics. The kaon signal can be seen in the negative $x_{\pi}$ tail, but one can also observe a small excess of tracks for $x_{\pi} \geq 4.5$. This 
excess has been investigated and is found to originate from the overlap of two tracks with only one track reconstructed. The effect is very small (a few $10^{-4}$ ) and only present for large positive $x_{\pi}$. It has been checked that excluding the region $x_{\pi} \geq 4.5$ does not affect the results of the kaon analysis.

To check $F_{\pi}\left(x_{\pi}\right)$ and get the final calibration, the $x_{\pi}$ distribution is fitted using the pion resolution function obtained from the $\omega$ sample, where the values for $\bar{x}_{\pi}(\pi)$ and $\sigma_{\pi}(\pi)$ are fixed at 0 and 1. A one-parameter fit of the kaon fraction yields $\chi^{2} / n d f=62.2 / 49$ (Fig. 4). A close inspection of the residuals between the data and the fitted function reveals a nearly slight antisymmetric deviation ${ }^{3}$ (see Fig. 4). A correction term for $F_{\pi}\left(x_{\pi}\right)$ is obtained by parametrizing the deviation in the range of $x_{\pi} \geq 0$, where the influence of kaons can be completely ignored. Assuming the correction is antisymmetric, a threeparameter fit is performed with the corrected $F_{\pi}\left(x_{\pi}\right)$ as input, giving the following values for the parameters: $f_{K}=(1.58 \pm 0.14) \%, \bar{x}_{\pi}(\pi)=0.006 \pm 0.005, \sigma_{\pi}(\pi)=0.998 \pm 0.004$ and $\chi^{2} / n d f=49.1 / 47$. The quality of the fit is improved, and the precise values for the pion calibration and the corrected $F_{\pi}\left(x_{\pi}\right)$ will be applied to all the following fits.

The difference $(0.12 \%)$ between the two kaon fractions found with the $\omega$-sample and the improved $F_{\pi}\left(x_{\pi}\right)$ shapes is treated as the systematic uncertainty related to the shape of the resolution function.

Since the kaon fraction is obtained in the three-parameter fit by allowing the calibration to float, the systematic uncertainties related to this effect are already included in the fitted error on $f_{K}$. In order to quote a systematic error from the calibration itself, a one-parameter fit for $f_{K}$ is performed with $\bar{x}_{\pi}(\pi)$ and $\sigma_{\pi}(\pi)$ fixed. The calibration error is obtained by unfolding the three- and one-parameter fit errors on $f_{K}$. This provides an uncertainty of $0.09 \%$ on the absolute error of the kaon fraction, which will be translated into the corresponding error for each channel.

Finally, to demonstrate the need for the kaon contribution, a two-parameter fit leaving the $x_{\pi}$ parameters free and the kaon content fixed at zero is done, yielding $\bar{x}_{\pi}(\pi)=-0.023 \pm 0.004, \sigma_{\pi}(\pi)=1.025 \pm 0.003$ and $\chi^{2} / n d f=125.1 / 48$. The much worse $\chi^{2}$ and the characteristic trend of the pion parameters, i.e. negative $\bar{x}_{\pi}(\pi)$ and larger $\sigma_{\pi}(\pi)$, validates the description with a genuine kaon contribution.

\subsection{Measured kaon fractions}

The kaon fractions are now extracted from the fits to the $3 h^{-} \nu_{\tau}$ and the $3 h^{-} \pi^{0} \nu_{\tau}$ final states, which are divided into same- and opposite-sign track samples. To reduce systematic uncertainties from $\tau$ decays without kaons, cuts on the well-measured hadronic mass are applied to enhance the relative kaon fraction. Only the $x_{\pi}$ of "kaon-assumed" tracks with $M\left(\pi^{+} K^{-} \pi^{-}\right) \leq m_{\tau}$ for the same-sign tracks and $M\left(K^{+} K^{-} \pi^{-}\right) \leq m_{\tau}$ for the oppositesign tracks are analysed. After applying these cuts, the pion tracks with available $\mathrm{dE} / \mathrm{dx}$ are reduced by $15 \%$ (same-sign) and $22 \%$ (opposite-sign) for the $3 h^{-} \nu_{\tau}$ mode and $8 \%$ (same-sign) and $12 \%$ (opposite-sign) for the $3 h^{-} \pi^{0} \nu_{\tau}$ mode, leaving the kaon contribution unaffected. As the consequence of these kinematic cuts, a fit for the pion-reduced sample gives: $f_{K}=(1.74 \pm 0.12) \%$ and $\chi^{2} / n d f=43.6 / 49$, in which the $x_{\pi}$ parameters are

\footnotetext{
${ }^{3}$ In fact the same effect for the resolution function is observed in one-prong decays, which is well determined using muon tracks and fitted to a function incorporating this feature [9].
} 


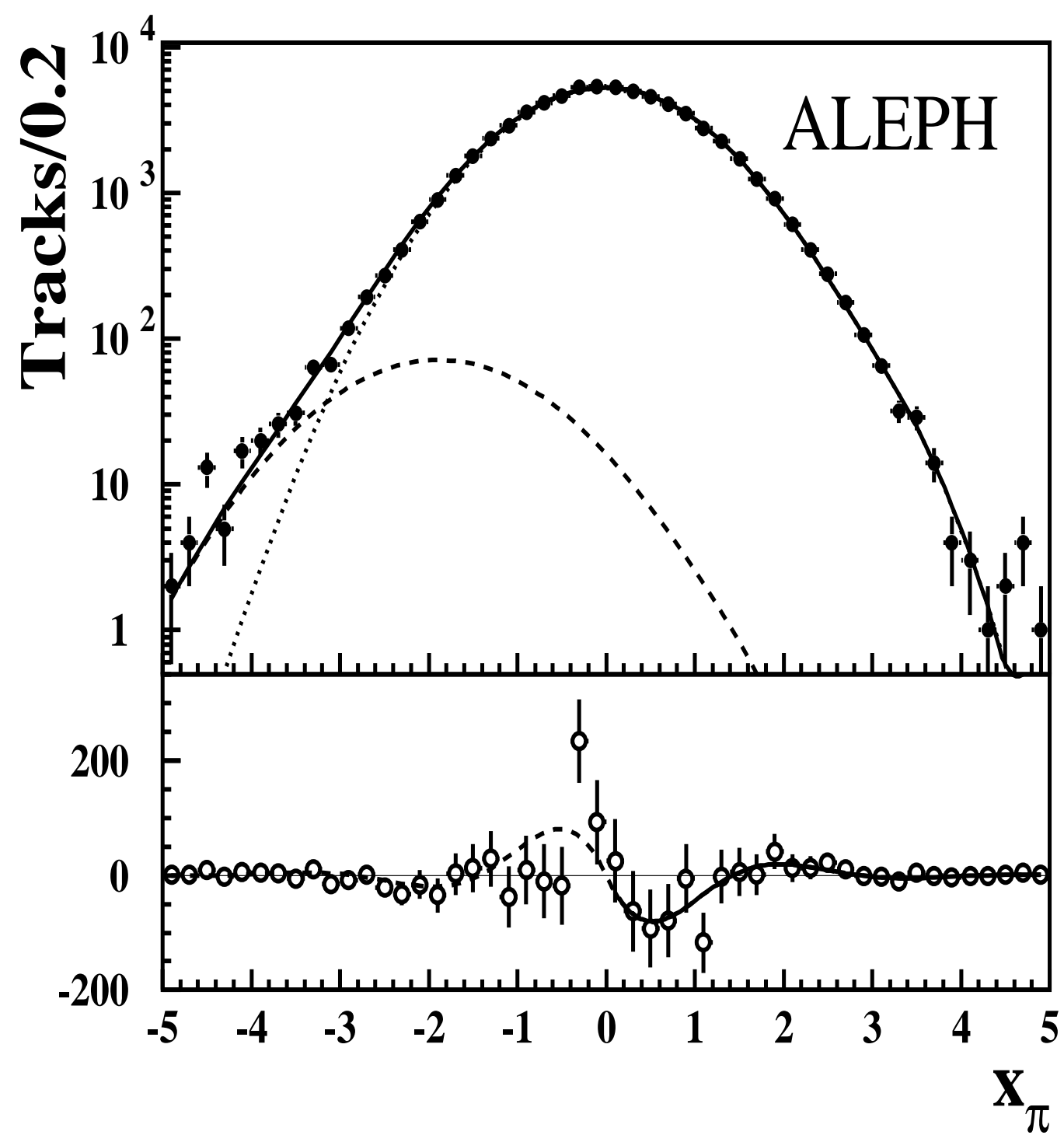

Figure 4: Fitted $x_{\pi}$ distribution for all tracks in $3 h^{-} \nu_{\tau}$ and $3 h^{-} \pi^{0} \nu_{\tau}$ (top). The dots with error bars correspond to data. The fit indicated by the solid curve is described in the text, while the dashed curve shows the fitted kaon contribution, The residuals between the data and the fit are shown in the bottom plot. The solid curve is the parametrization for $x_{\pi} \geq 0$ and the dashed curve shows the expected deviation on the negative side assuming antisymmetry. 


\begin{tabular}{|l|c|c|c|c|}
\hline \hline Mode & \multicolumn{2}{|c|}{$3 h^{-} \nu_{\tau}$} & \multicolumn{2}{c|}{$3 h^{-} \pi^{0} \nu_{\tau}$} \\
\hline Sign & Same & Opposite & Same & Opposite \\
\hline $\bar{x}_{\pi}(K)$ & $-1.914 \pm 0.016$ & $-1.875 \pm 0.022$ & $-1.774 \pm 0.022$ & $-1.729 \pm 0.032$ \\
\hline$\sigma_{\pi}(K)$ & $1.080 \pm 0.012$ & $1.093 \pm 0.017$ & $1.097 \pm 0.016$ & $1.035 \pm 0.025$ \\
\hline $\bar{x}_{\pi}(\pi)$ & 0.006 (fixed) & 0.006 (fixed) & 0.006 (fixed) & 0.006 (fixed) \\
\hline$\sigma_{\pi}(\pi)$ & 0.998 (fixed) & 0.998 (fixed) & 0.998 (fixed) & 0.998 (fixed) \\
\hline$f_{K}(\%)$ & $2.11 \pm 0.18$ & $1.62 \pm 0.25$ & $1.24 \pm 0.30$ & $0.80 \pm 0.41$ \\
\hline$N_{K}$ & $597 \pm 51$ & $229 \pm 35$ & $120 \pm 29$ & $38 \pm 19$ \\
\hline$\chi^{2} / n d f$ & $59.0 / 48$ & $46.9 / 42$ & $46.3 / 41$ & $50.1 / 39$ \\
\hline$N_{K}(q \bar{q})$ & $10 \pm 3$ & $6 \pm 2$ & $9 \pm 3$ & $4 \pm 2$ \\
\hline \hline
\end{tabular}

Table 2: Results obtained from the fits to $x_{\pi}$ distributions. The fits take into account both kaon and pion contributions. The kaon parameters $\bar{x}_{\pi}(K)$ and $\sigma_{\pi}(K)$ are determined from Monte Carlo and fixed in the fits to data. The errors of these parameters from Monte Carlo statistics are translated into systematic uncertainties on the branching ratios due to Monte Carlo statistics. The definition for each quantity can be found in the text.

fixed at 0.006 and 0.998 . Obviously, the $f_{K}$ is increased. Also, a Monte Carlo study shows that these kinematic cuts eliminate about $50 \%$ of kaon tracks with a $\mathrm{dE} / \mathrm{dx}$ measurement from the $q \bar{q}$ background. The remaining $q \bar{q}$ background $N_{K}(q \bar{q})$, normalized to the same statistics as data is shown in Table 2.

The final kaon fraction for each sample is obtained from the one-parameter fit in which the $x_{\pi}(\pi)$ parameters are fixed at the values obtained from the global calibration described above. Fig. 5 shows the fit results for the same-sign tracks and the opposite-sign tracks in the $3 h^{-} \nu_{\tau}$ and the $3 h^{-} \pi^{0} \nu_{\tau}$ modes, respectively. The fit results for each sample are summarized in Table 2.

Because a pure kaon sample with the same environment of overlapping track cannot be isolated directly from the data, the kaon parameters can only be estimated from the Monte Carlo simulation (with the input of the measured $F_{\pi}\left(x_{\pi}\right)$ distribution), which shows the expected correlation between $\bar{x}_{\pi}(K)$ and the track momentum (see Fig. 6). The kaon momentum spectrum in $\tau$ lepton decays depends somewhat on the dynamics. To check the kaon momentum distribution in data, a one-parameter fit in momentum slices is also performed in the $3 h^{-} \nu_{\tau}$ mode. The corresponding number of kaons in each momentum slice is shown in Fig. 7. The sum of all momentum slices gives $580 \pm 50$ and $228 \pm 35$ for the same- and opposite-sign kaons, in agreement with the total amount of kaons obtained from the fit (Table 2) to the whole momentum range $(2-45 \mathrm{GeV} / c)$. This indicates an agreement between data and simulation for the kaon momentum distribution (Fig. 7), although there is some tendency for a slightly softer spectrum in data for same-sign kaons in $3 h^{-} \nu_{\tau}$ decays. It is also seen in Fig. 7 that the kaon momentum spectrum is found to be harder than for pions.

Finally, in order to select $K^{-} K^{+} \pi^{-} \nu_{\tau}$ and $K^{-} \pi^{+} \pi^{-} \nu_{\tau}$ samples for the purpose of studying the decay dynamics, a kaon identification estimator is defined using the 


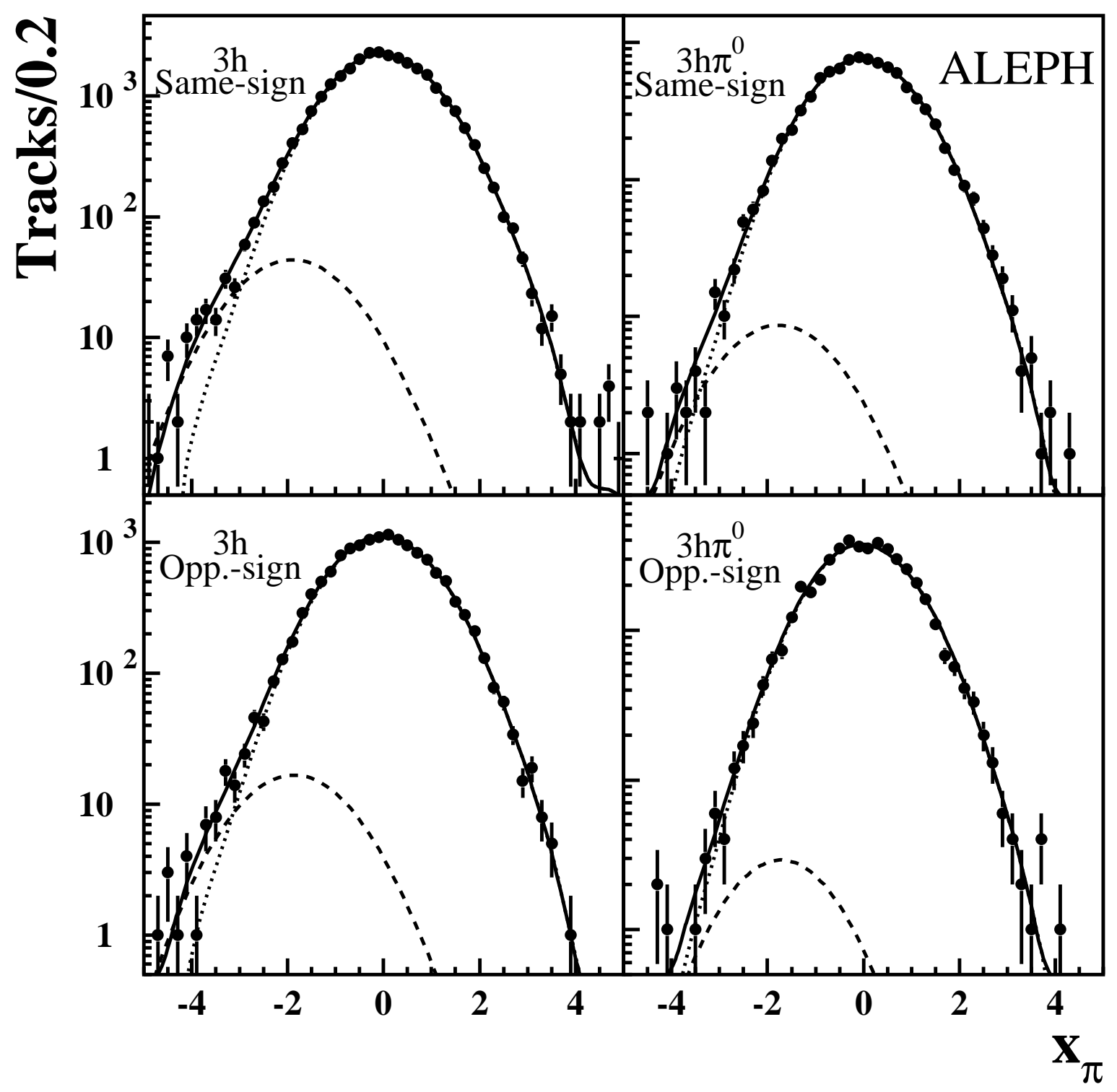

Figure 5: Fitted $x_{\pi}$ distributions for the pion-reduced samples. The dots with error bars correspond to data. The fits indicated by solid curves are described in the text. The dashed curves show the fitted $K$ components while the dotted curves represent the $\pi$ contributions. 


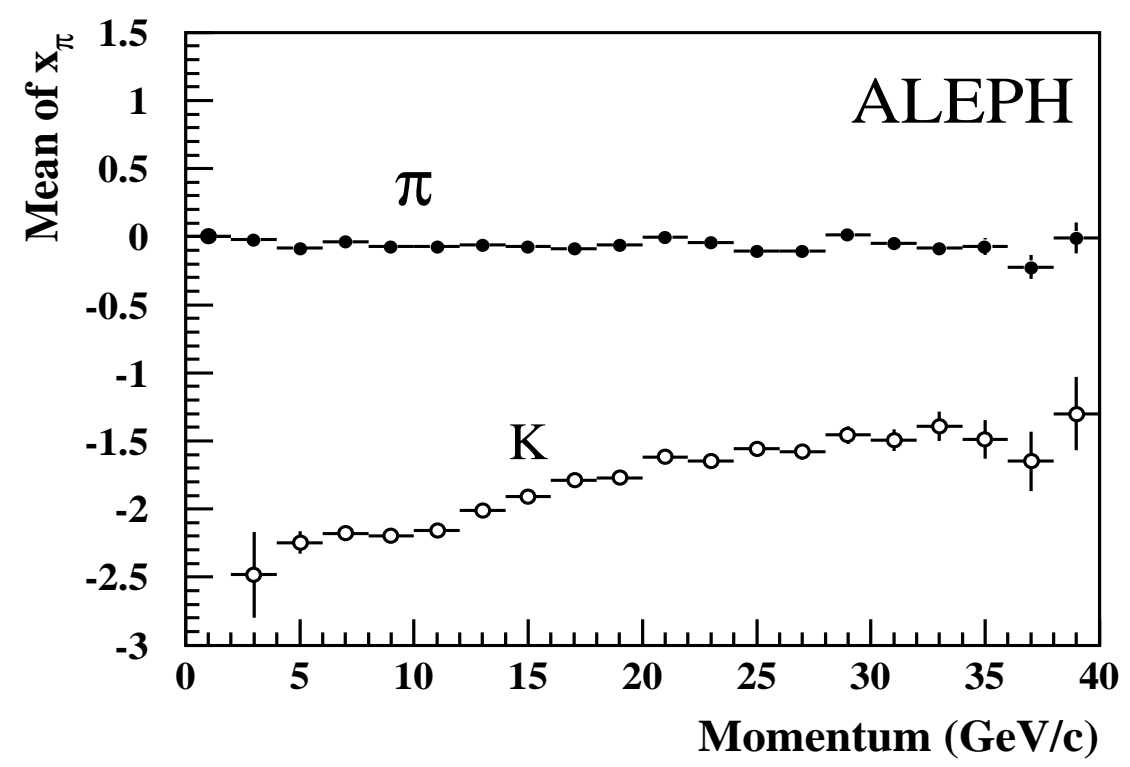

Figure 6: The $K / \pi$ separation in $x_{\pi}$ as a function of momentum for $3 h^{-} \nu_{\tau}$ Monte Carlo samples. The closed circles correspond to pions and the open circles to kaons.

recalibrated $\mathrm{dE} / \mathrm{dx}$ measurement

$$
P_{K}=\frac{W_{K}(R)}{W_{\pi}(R)+W_{K}(R)},
$$

where $W_{\pi, K}(R)$ is the probability for the track to be a pion or a kaon with a given $\mathrm{dE} / \mathrm{dx}$ measurement $R$. Correspondingly, one can define a pion probability $P_{\pi}=1-P_{K}$. The $K / \pi$ separation is found to be momentum-dependent, decreasing from $2.3 \sigma$ at $5 \mathrm{GeV} / c$ to $1.5 \sigma$ at $40 \mathrm{GeV} / c$ (Fig. 6).

\section{Measurement of the branching ratios}

All branching ratios are derived using the observed number of $\tau$ decay candidates in each channel and the estimate of the background from other $\tau$ decays and non- $\tau$ sources ( $Z$ decays to hadrons), according to

$$
B_{i}=\frac{N_{K}-N_{K}(q \bar{q})}{2 N_{\tau \tau} \epsilon_{i}}-\sum_{j \neq i} B_{j} \frac{\epsilon_{j}}{\epsilon_{i}},
$$

where $B_{i}$ and $B_{j}$ are the branching ratios for the signal and the feedthrough $\tau$ backgrounds. The corresponding efficiencies are $\epsilon_{i}$ and $\epsilon_{j}$, estimated for the same- or opposite-sign samples (Table 1). $N_{\tau \tau}, N_{K}$ and $N_{K}(q \bar{q})$ are the total number of produced $\tau$ pairs, the number of measured kaons and the expected $q \bar{q}$ background, respectively. 


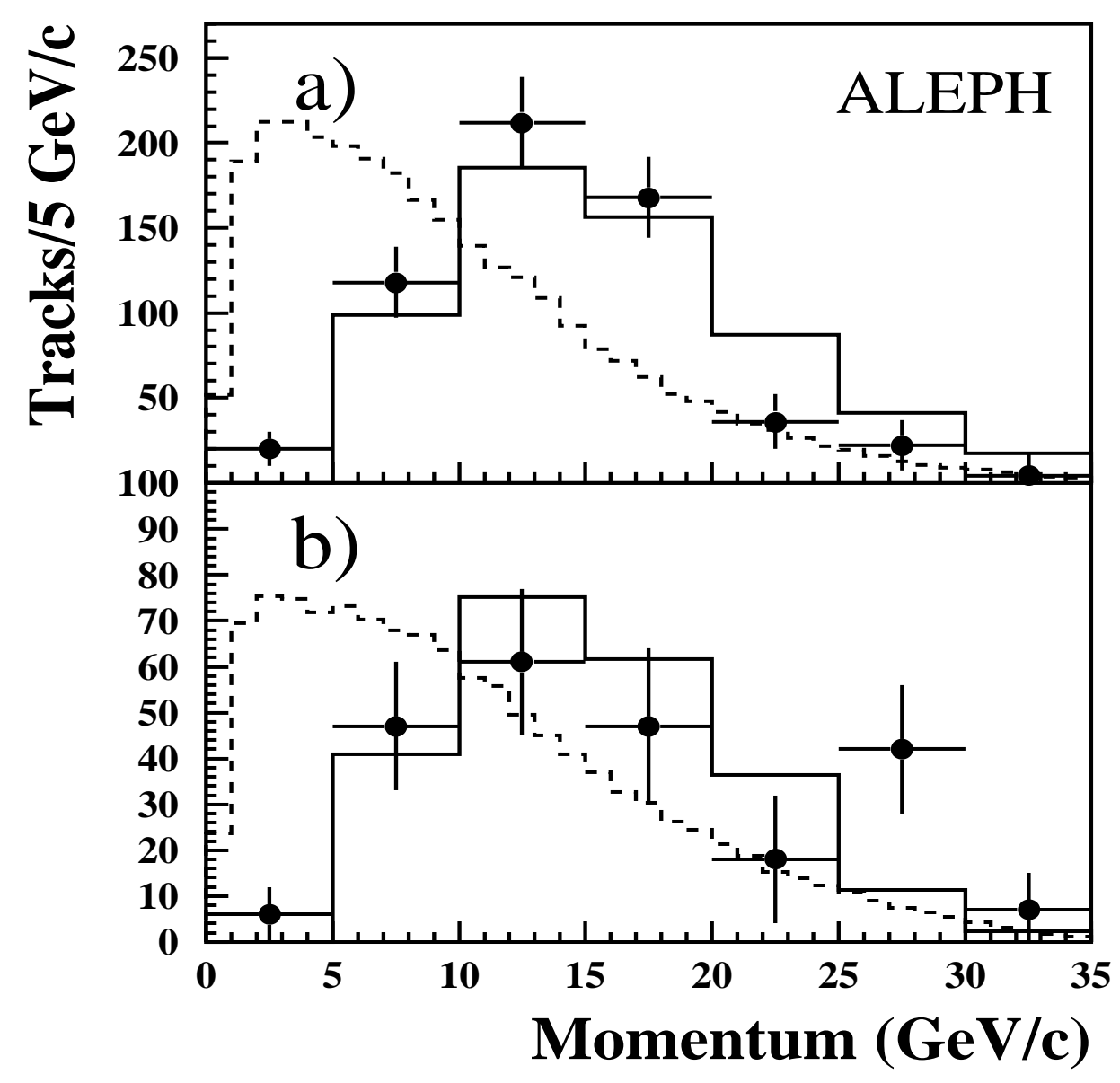

Figure 7: The distributions of kaon momenta for a) same-sign kaons and b) oppositesign kaons in $3 h^{-} \nu_{\tau}$. The dots with error bars correspond to the fitted data and the solid histograms are the Monte Carlo expectations following the model of Ref [4]. The dashed histograms are the expected distributions of pion momenta. 


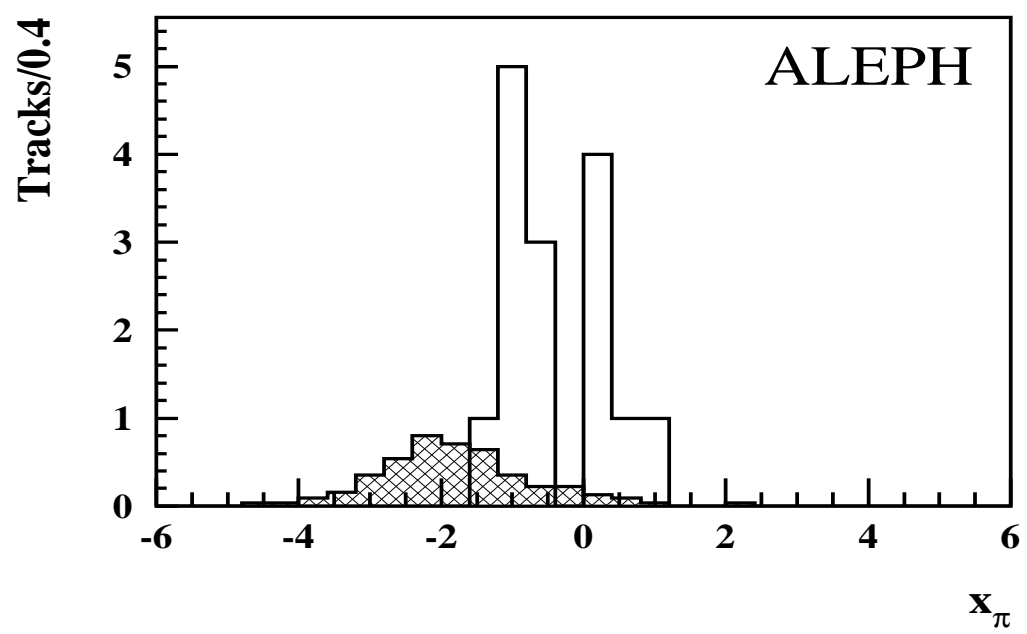

Figure 8: The $x_{\pi}$ distribution for the selected same-sign tracks in the study of $\tau^{-} \rightarrow K^{-} K^{+} K^{-} \nu_{\tau}$ after $K^{+}$and $K^{-}$cuts. Data are shown in the unhatched histogram while the expected kaon distribution is shown in the hatched histogram.

\subsection{Search for $\tau^{-} \rightarrow K^{-} K^{+} K^{-} \nu_{\tau}$}

The $K^{-} K^{+} K^{-} \nu_{\tau}$ branching ratio is expected to be very small, because of phase-space and Cabibbo suppression. To select this channel, track momenta are required to exceed $10 \mathrm{GeV} / c$ and the invariant mass of the $K^{-} K^{+} K^{-}$system must be smaller than $m_{\tau}$. To reduce the pion background, the $x_{\pi}$ of the opposite-sign track is required to be less than zero, rejecting half of the remaining pions and keeping all opposite-sign kaons, and one of the same-sign tracks must satisfy $x_{\pi} \leq-1.5$, keeping slightly more than half of the kaons. No significant kaon signal appears in the $x_{\pi}$ distribution for the third track (same-sign) (see Fig. 8). According to the Monte Carlo simulation, the acceptance for $K^{-} K^{+} K^{-} \nu_{\tau}$ is $7.1 \%$ and from a maximum likelihood fit of the $x_{\pi}$ distribution an upper limit is obtained

$$
B\left(\tau^{-} \rightarrow K^{-} K^{+} K^{-} \nu_{\tau}\right)<1.9 \times 10^{-4} \quad(95 \% \text { C.L. })
$$

In the following analysis, this contribution will be neglected, since the limit on the branching ratio is significantly smaller than the uncertainties achieved in the measurement of the other channels, and furthermore the expected value from the model [4] is no more than $10^{-6}$.

\subsection{The branching ratios for $\tau^{-} \rightarrow K^{-} K^{+} \pi^{-}\left(\pi^{0}\right) \nu_{\tau}$}

Two methods are employed for measuring the branching ratios for the $K^{-} K^{+} \pi^{-}\left(\pi^{0}\right) \nu_{\tau}$ modes. The first method is based on the number of the opposite-sign $K^{\prime}$ 's. Only the $K^{-} K^{+} \pi^{-} \nu_{\tau}$ and the $K^{-} K^{+} \pi^{-} \pi^{0} \nu_{\tau}$ can produce opposite-sign $K$ 's. The numbers of kaons from the one-parameter fit are summarized in Table 2. The feedthrough from $\tau$ and non- $\tau$ 


\begin{tabular}{|c|c|c|}
\hline \hline Mode & $K^{-} K^{+} \pi^{-} \nu_{\tau}$ & $K^{-} K^{+} \pi^{-} \pi^{0} \nu_{\tau}$ \\
\hline Cuts (opp.) & $x_{\pi} \leq-2$ and $p \geq 5 \mathrm{GeV} / c$ & $x_{\pi} \leq-1.75$ and $p \geq 6 \mathrm{GeV} / c$ \\
\hline Cuts (same) & $p \geq 5 \mathrm{GeV} / c$ & $p \geq 6 \mathrm{GeV} / c$ \\
\hline$f_{K}$ (same) $\%$ & $26.0 \pm 3.7$ & $12.9 \pm 5.5$ \\
\hline$N_{K}$ (same) & $82 \pm 12$ & $14 \pm 6$ \\
\hline Background & $14 \pm 4$ & $1 \pm 1$ \\
\hline Signal & $68 \pm 14$ & $13 \pm 6$ \\
\hline Efficiency (\%) & $10.48 \pm 0.43$ & $3.52 \pm 0.20$ \\
\hline B $\left(10^{-3}\right)$ & $1.62 \pm 0.33$ & $0.92 \pm 0.42$ \\
\hline \hline
\end{tabular}

Table 3: Summary of all quantities obtained by tagging an opposite-sign kaon and fitting to the $x_{\pi}$ for same-sign tracks. Errors are statistical only.

backgrounds is subtracted with respect to the corresponding efficiencies in Table 1, and the branching ratios are obtained by combining the $x_{\pi}$ fits for the opposite-sign tracks in the $3 h^{-} \nu_{\tau}$ and the $3 h^{-} \pi^{0} \nu_{\tau}$ samples, yielding

$$
\begin{gathered}
\left.B\left(\tau^{-} \rightarrow K^{-} K^{+} \pi^{-} \nu_{\tau}\right)=\left(1.63 \pm 0.27_{\text {stat. }}\right) \times 10^{-3} \quad \text { (opposite-sign fit }\right) \\
\left.B\left(\tau^{-} \rightarrow K^{-} K^{+} \pi^{-} \pi^{0} \nu_{\tau}\right)=\left(0.59 \pm 0.41_{\text {stat. }}\right) \times 10^{-3} \quad \text { (opposite-sign fit }\right) .
\end{gathered}
$$

Since there are two kaons in the final state, a second method for determining the branching ratios is to tag the opposite-sign $K$ 's with a cut on the $x_{\pi}(K)$ and fit the $x_{\pi}$ distribution for same-sign tracks. In this way, the background from the $\pi^{-} \pi^{+} \pi^{-}\left(\pi^{0}\right) \nu_{\tau}$ and the $K^{-} \pi^{+} \pi^{-}\left(\pi^{0}\right) \nu_{\tau}$ channels is strongly suppressed and the kaon signal from the $K^{-} K^{+} \pi^{-}\left(\pi^{0}\right) \nu_{\tau}$ is enhanced as shown in Fig. 9. All cuts, related quantities and branching ratios are given in Table 3. The small statistical correlation $(\sim 20 \%)$ between the two values is estimated using a toy Monte Carlo. The results are combined and give

$$
\begin{gathered}
B\left(\tau^{-} \rightarrow K^{-} K^{+} \pi^{-} \nu_{\tau}\right)=\left(1.63 \pm 0.21_{\text {stat. }}\right) \times 10^{-3} \quad \text { (average) } \\
\left.B\left(\tau^{-} \rightarrow K^{-} K^{+} \pi^{-} \pi^{0} \nu_{\tau}\right)=\left(0.75 \pm 0.29_{\text {stat. }}\right) \times 10^{-3} \quad \text { (average }\right) .
\end{gathered}
$$

\subsection{The branching ratios for $\tau^{-} \rightarrow K^{-} \pi^{+} \pi^{-}\left(\pi^{0}\right) \nu_{\tau}$}

The measurements of these two channels are based only on the $x_{\pi}$ fits for same-sign tracks. Many feedthrough $\tau$ and non- $\tau$ backgrounds can produce same-sign kaons. Besides the $K^{-} K^{+} \pi^{-}$and the $K^{-} K^{+} \pi^{-} \pi^{0}$ modes, the $K^{0} K^{-}$and $K^{0} K^{-} \pi^{0}$ modes are also found to give a non-negligible contribution to the same-sign kaons. This effect is reflected in the values for the efficiencies listed in Table 1. Subtracting the feedthrough background from the $K^{-} K^{+} \pi^{-}\left(\pi^{0}\right) \nu_{\tau}$ and the $K^{-} K^{0}\left(\pi^{0}\right) \nu_{\tau}$ (the second branching ratios taken from the world averages [12]) gives the measurements of the $K^{-} \pi^{+} \pi^{-}\left(\pi^{0}\right) \nu_{\tau}$

$$
B\left(\tau^{-} \rightarrow K^{-} \pi^{+} \pi^{-} \nu_{\tau}\right)=\left(2.14 \pm 0.37_{\text {stat. }}\right) \times 10^{-3},
$$




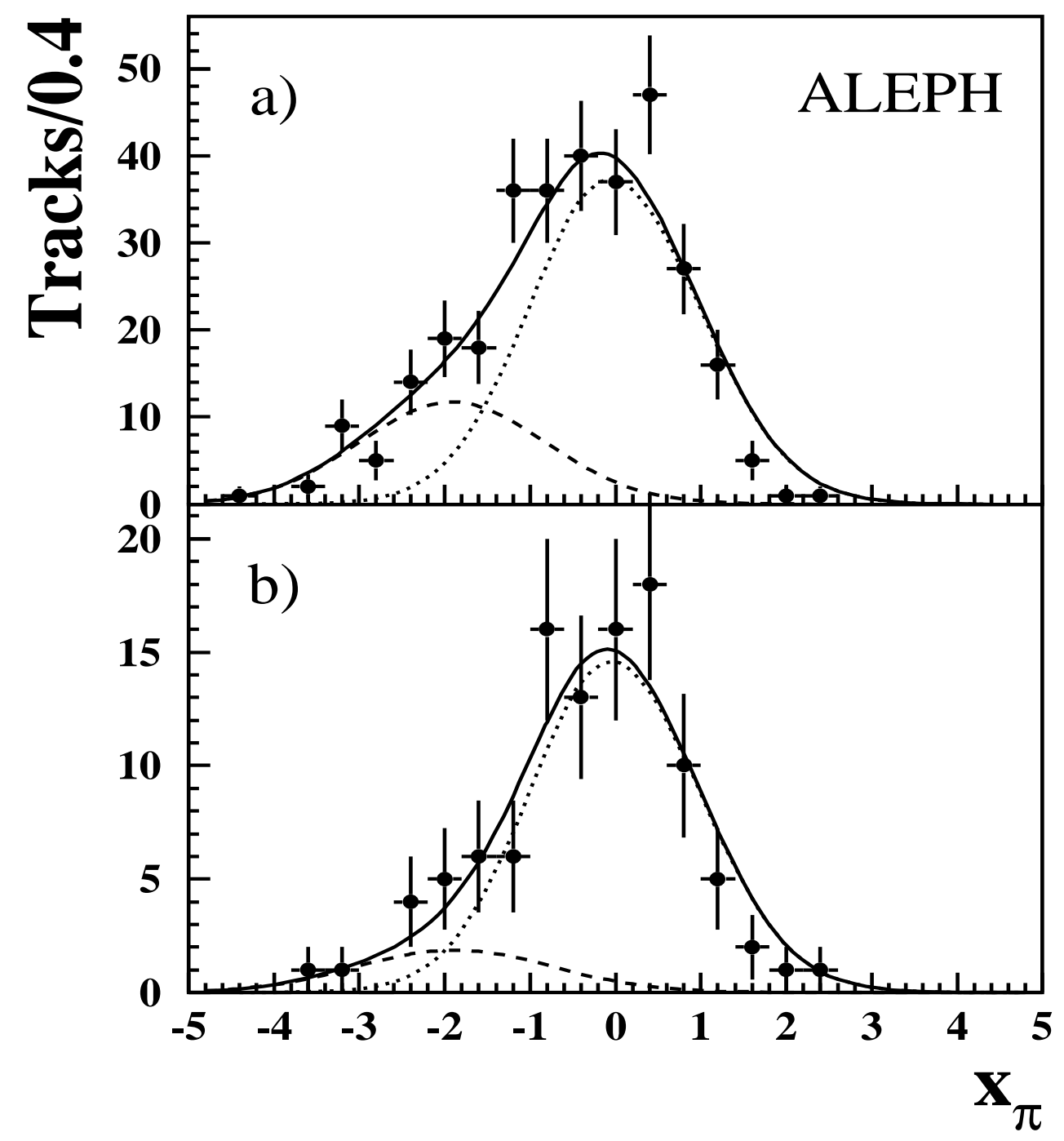

Figure 9: Fitted $x_{\pi}$ distribution for same-sign tracks in a) $\tau^{-} \rightarrow K^{-} K^{+} \pi^{-} \nu_{\tau}$ and b) $\tau^{-} \rightarrow K^{-} K^{+} \pi^{-} \pi^{0} \nu_{\tau}$, after tagging of the opposite-sign kaon. The plots with error bars correspond to data, the solid curves are the sum and the dashed curves show the fitted $K$ components while the dotted curves indicate the $\pi$ contributions. 


\begin{tabular}{|c|c|c|c|c|}
\hline \hline Source & $K^{-} \pi^{+} \pi^{-}$ & $K^{-} K^{+} \pi^{-}$ & $K^{-} \pi^{+} \pi^{-} \pi^{0}$ & $K^{-} K^{+} \pi^{-} \pi^{0}$ \\
\hline Selection efficiency & 1.0 & 1.0 & 1.9 & 1.9 \\
\hline $\mathrm{dE} / \mathrm{dx}$ efficiency & 4.2 & 4.5 & 5.6 & 4.7 \\
\hline $\mathrm{dE} / \mathrm{dx}$ calibration & 5.3 & 3.5 & 12.7 & 7.0 \\
\hline$F_{\pi}\left(x_{\pi}\right)$ shape & 7.1 & 4.7 & 16.9 & 9.3 \\
\hline$x_{\pi}(K)$ & 5.5 & 3.9 & 10.1 & 5.7 \\
\hline$\tau$ background & 4.2 & 3.6 & 10.4 & 5.4 \\
\hline$q \bar{q}$ background & 1.1 & 1.7 & 7.1 & 7.1 \\
\hline M.C. statistics (eff.) & 2.4 & 1.6 & 4.6 & 3.6 \\
\hline Dynamics (eff.) & 5.4 & 4.1 & 10 & 10 \\
\hline Total & 13.5 & 10.3 & 29.4 & 19.6 \\
\hline \hline
\end{tabular}

Table 4: Summary of systematic errors. All are relative in percent.

$$
B\left(\tau^{-} \rightarrow K^{-} \pi^{+} \pi^{-} \pi^{0} \nu_{\tau}\right)=\left(0.61 \pm 0.39_{\text {stat. }}\right) \times 10^{-3} .
$$

The errors quoted in (12) and (13) are dominated by the statistical uncertainties on the number of same-sign kaons from the fit. They also include the statistical uncertainty from the subtraction of the $K^{-} K^{+} \pi^{-}\left(\pi^{0}\right) \nu_{\tau}$ channels (10) and (11), thereby introducing a small anticorrelation of $-14 \%$ for results (10) and (12).

\section{Sources of systematic errors}

Several sources of systematic errors have been considered: selection, charged track overlap, $\mathrm{dE} / \mathrm{dx}$ calibration, $\mathrm{dE} / \mathrm{dx}$ response for pions, the $x_{\pi}(K)$ parameters, feedthrough backgrounds, Monte Carlo statistics and the dynamics of hadronic $\tau$ decays. All systematic errors are summarized in Table 4 and the corresponding discussion of the individual sources is given below.

\subsection{Selection efficiency}

The systematic uncertainties coming from the general $\tau$-pair selection and classification criteria have been studied in Ref [8]. The additional requirement of exactly three charged tracks somewhat increases the systematic uncertainty in the selection efficiency. By comparing the data and the Monte Carlo distributions in the hemispheres with two, three and four charged tracks (after removal of identified electrons from $\gamma$ conversions), it is found that the efficiency to reconstruct exactly three tracks is slightly overestimated in the Monte Carlo by a factor $1.009 \pm 0.005$. Also detailed studies [8] have shown that the simulation underestimates the fraction of fake photons produced by hadronic interactions in ECAL and by the splitting of electromagnetic showers. Consequently, the selection efficiency for $3 h^{-}$final states is overestimated by a factor of $1.019 \pm 0.005$. For the $3 h^{-} \pi^{0}$ final states, the effect is slightly reduced since the loss of decays caused by the validation of an extra photon is partially compensated by the accidental reconstruction of a $\pi^{0}$ due to the presence of a fake photon, giving a corresponding factor of $1.011 \pm 0.006$. 
These correction factors are applied to the selection efficiency that is estimated with Monte Carlo. The corresponding errors are taken as systematic uncertainties. Total uncertainties of $1.0 \%$ and $1.9 \%$ for $3 h^{-} \nu_{\tau}$ and $3 h^{-} \pi^{0} \nu_{\tau}$ are estimated.

\subsection{Efficiency of tracks with $\mathrm{dE} / \mathrm{dx}$}

The efficiency from requiring a minimum number of $\mathrm{dE} / \mathrm{dx}$ samples is affected by the overlap of charged tracks. This effect depends on the simulation of the $\mathrm{dE} / \mathrm{dx}$ measurement and on the description of the decay dynamics. The $\mathrm{dE} / \mathrm{dx}$ sample efficiencies for data and Monte Carlo in the total three-prong sample agree (see Section 4). Unfortunately, a direct comparison cannot be made for the channels with kaons since they are only separated statistically from the dominant $3 \pi\left(\pi^{0}\right)$ decay modes. Nonetheless, an indirect test can be performed.

To monitor any systematic effect between data and Monte Carlo, the correlation of the number of samples with hadronic mass is exploited. Smaller hadronic masses are correlated with smaller opening angles, hence larger track overlap. A large variation in efficiency is observed over the $3 \pi$ mass range; however the ratio of the data to the Monte Carlo efficiencies is consistent with unity throughout (Fig. 10). A linear fit is applied to describe a possible systematic effect: slopes of $(-0.063 \pm 0.041)\left(\mathrm{GeV} / c^{2}\right)^{-1}$ and $(0.026 \pm 0.059)\left(\mathrm{GeV} / c^{2}\right)^{-1}$ are found for same-sign and opposite-sign tracks respectively. Corresponding systematic uncertainties for the $\mathrm{dE} / \mathrm{dx}$ track efficiency in every channel studied are obtained from this fit, scaled to the Monte Carlo efficiency specific to that channel (Table 4).

\subsection{Pion $\mathrm{dE} / \mathrm{dx}$ calibration}

The uncertainty from the pion calibration is obtained from the three-parameter fit (discussed in Section 6.2) compared to the one-parameter fit, leaving the $\bar{x}_{\pi}(\pi)$ and $\sigma_{\pi}(\pi)$ parameters fixed at their central values. The uncertainty of $0.09 \%$ on the absolute kaon fraction is then applied in a relative way to all channels (Table 4) since those with a small kaon contribution are more affected by pion calibration uncertainty. In this respect, the measurement of the $K^{-} K^{+} \pi^{-}\left(\pi^{0}\right)$ channels from the " $K^{+} K^{-}$" fits given in Table 3 and in Fig. 9 are not significantly affected by this uncertainty.

\section{4 $\mathrm{dE} / \mathrm{dx}$ response for pions}

Corrections for the pion resolution function $F_{\pi}\left(x_{\pi}\right)$ have been established using the full three-prong sample $\left(3 h^{-}\right.$and $\left.3 h^{-} \pi^{0}\right)$. The uncertainty on the kaon fraction from an imperfect knowledge of the shape of $F_{\pi}\left(x_{\pi}\right)$ has been estimated from the full effect of the correction applied. An uncertainty of $0.12 \%$ is found for the absolute kaon fraction which is then propagated to all measurements (Table 4). 


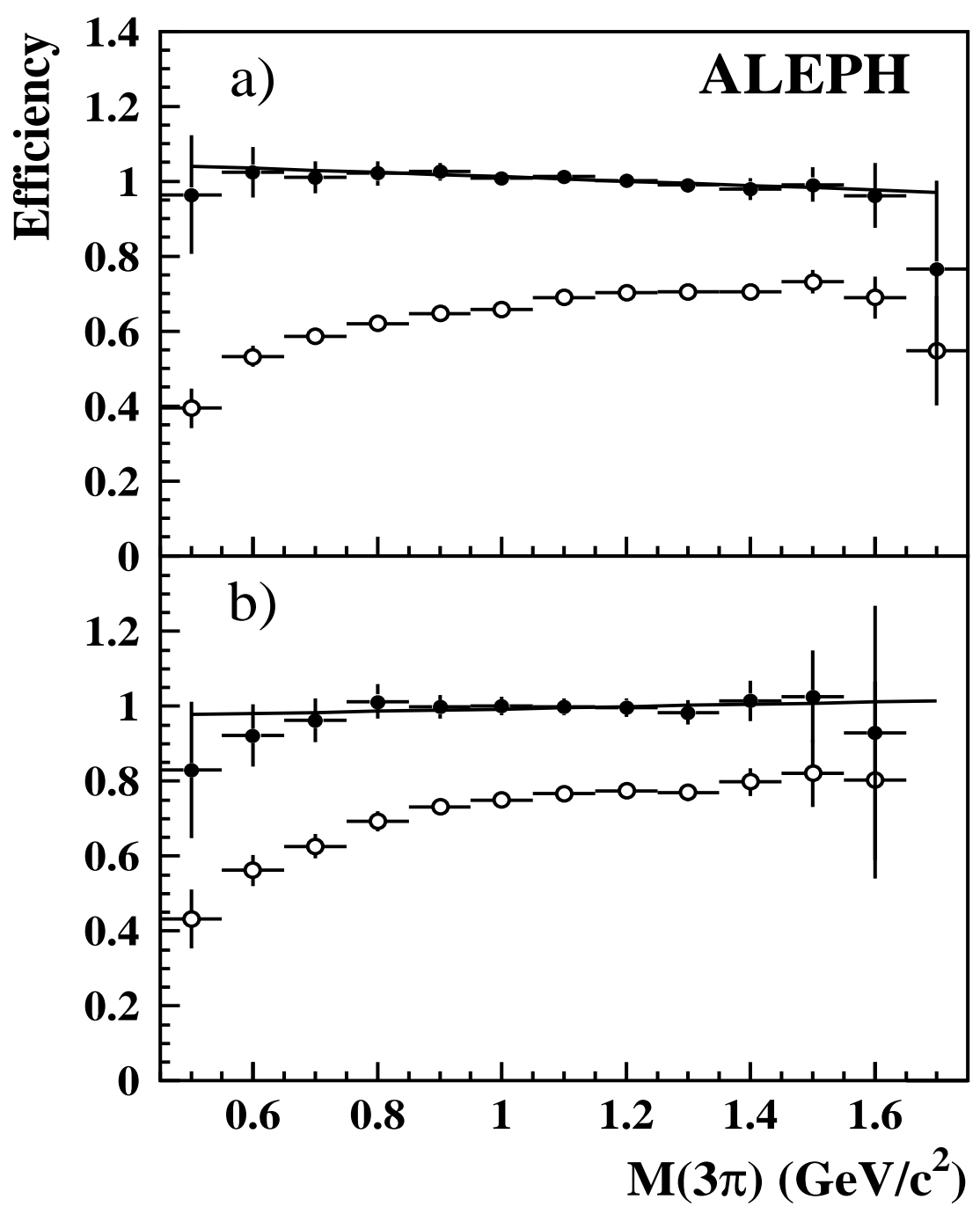

Figure 10: The ratio of the data to the Monte Carlo efficiencies for $d E / d x$ measurement. Results from $3 h^{-}$and $3 h^{-} \pi^{0}$ are combined. a) same-sign and b) opposite-sign tracks are studied separately. The linear fit performed is consistent within statistics with a constant value of one. The open circles show the behaviour of the corresponding absolute efficiencies in data. 


\subsection{Kaon $\mathrm{dE} / \mathrm{dx}$}

The systematic uncertainties associated with the kaon parameters $\bar{x}_{\pi}(K)$ and $\sigma_{\pi}(K)$ arise from two parts: the imperfect simulation of the track overlap and the uncertainties from the modelling of the final states in the Monte Carlo.

By comparing the mean number of samples between data and Monte Carlo, one finds $186.9 \pm 0.4$ and $196.0 \pm 0.6$ for the same- and opposite-sign tracks in the data, and $184.9 \pm 0.2$ and $194.8 \pm 0.3$ in the Monte Carlo, respectively. The differences, with one standard deviation added, yielding the values of 2.4 and 1.9 for same- and opposite-sign tracks, are treated as a systematic effect due to an imperfect simulation. These values are translated into offsets for $\bar{x}_{\pi}(K)$ of 0.0142 and 0.0125 for the same- and opposite-sign cases. The relevant uncertainties are found to be $1.5 \%\left(K^{-} \pi^{+} \pi^{-} \nu_{\tau}\right), 0.9 \%\left(K^{-} K^{+} \pi^{-} \nu_{\tau}\right)$, $2.4 \%\left(K^{-} \pi^{+} \pi^{-} \pi^{0} \nu_{\tau}\right)$ and $0.9 \%\left(K^{-} K^{+} \pi^{-} \nu_{\tau}\right)$.

The errors of the $x_{\pi}(K)$ parameters come from both Monte Carlo statistics and modelling of the final state dynamics. The errors from the statistical uncertainties of the $x_{\pi}(K)$ parameters listed in Table 2 are obtained by studying the deviation of the kaon fraction within one standard deviation of the $x_{\pi}(K)$ parameters, giving $3.3 \%$ $\left(K^{-} \pi^{+} \pi^{-} \nu_{\tau}\right), 2.7 \%\left(K^{-} K^{+} \pi^{-} \nu_{\tau}\right), 7.2 \%\left(K^{-} \pi^{+} \pi^{-} \pi^{0} \nu_{\tau}\right)$ and $4.2 \%\left(K^{-} K^{+} \pi^{-} \nu_{\tau}\right)$. Since the decay dynamics can also affect the $x_{\pi}(K)$ parameters through the track overlap, this effect is investigated through the correlation between the $x_{\pi}(K)$ parameters and the total hadronic mass. The uncertainties are estimated to be $4.1 \%\left(K^{-} \pi^{+} \pi^{-} \nu_{\tau}\right), 2.6 \%$ $\left(K^{-} K^{+} \pi^{-} \nu_{\tau}\right), 6.6 \%\left(K^{-} \pi^{+} \pi^{-} \pi^{0} \nu_{\tau}\right)$ and $3.8 \%\left(K^{-} K^{+} \pi^{-} \nu_{\tau}\right)$. The final errors due to the $x_{\pi}(K)$ uncertainties are obtained by adding the above contributions in quadrature and expressed as relative errors on the branching ratios.

\subsection{Background subtraction}

The $q \bar{q}$ non- $\tau$ background is found to be small for the $3 h^{-} \nu_{\tau}$ samples: $K^{-} \pi^{+} \pi^{-} \nu_{\tau}(1.7 \%)$ and $K^{-} K^{+} \pi^{-} \nu_{\tau}(1.2 \%)$, but it is more significant for the $3 h^{-} \pi^{0} \nu_{\tau}$ samples: $K^{-} \pi^{+} \pi^{-} \pi^{0} \nu_{\tau}$ (7.1\%) and $K^{-} K^{+} \pi^{-} \pi^{0} \nu_{\tau}(7.1 \%)$

When evaluating the feedthrough $\tau$ background contributions to the studied channels, the uncertainties in the branching ratios of these backgrounds are taken into account. The background subtraction depends on the corresponding efficiencies listed in Table 1 and the uncertainties in the relevant branching ratios. For the $K^{0} K^{-} \nu_{\tau}$ and the $K^{0} K^{-} \pi^{0} \nu_{\tau}$ channels, the values of $(1.55 \pm 0.28) \times 10^{-3}$ and $(1.38 \pm 0.32) \times 10^{-3}$ are taken from Ref [12]. The uncertainties in the feedthrough resulting from $\pi^{0}$ reconstruction and simulation of fake photons are estimated following the studies detailed in Ref [8]. No additional uncertainty is introduced in the background from channels differing by charged particle identification as they are measured simultaneously in this analysis. On the whole, the systematic uncertainties are found to be smaller for the channels producing an opposite-sign kaon: $3.6 \%\left(K^{-} K^{+} \pi^{-} \nu_{\tau}\right)$ and $5.4 \%\left(K^{-} K^{+} \pi^{-} \pi^{0} \nu_{\tau}\right)$. As a result of involving more feedthrough background, the uncertainties for the $K^{-} \pi^{+} \pi^{-} \nu_{\tau}$ and the $K^{-} \pi^{+} \pi^{-} \pi^{0} \nu_{\tau}$ channels are larger: $4.2 \%$ and $10.4 \%$. 


\subsection{Monte Carlo statistics and decay dynamics}

The uncertainties due to limited Monte Carlo statistics for the determination of the efficiency matrix are listed in Table 1. Due to the lack of knowledge of the dynamics of some of the hadronic $\tau$ decays, the efficiency matrix is found to be sensitive to the shape of the hadronic invariant mass distribution, which is model-dependent. A study of the efficiency as a function of the hadronic mass (which is the dominant factor) is done for each channel. Estimation of the uncertainties is based on the comparison of the resonance structure between data and Monte Carlo. The resonance structures are known from data (see Section 9) for the $K^{-} K^{+} \pi^{-} \nu_{\tau}\left(+70 \mathrm{MeV} / c^{2}\right.$ mass shift and $K^{*} K$ dominance) and the $K^{-} \pi^{+} \pi^{-} \nu_{\tau}\left(-100 \mathrm{MeV} / c^{2}\right.$ with a mixture of $K_{1}(1270)$ and $\left.K_{1}(1400)\right)$ modes. The corresponding efficiencies are directly estimated by using the corrected model. The associated systematic errors due to the uncertainties in the model corrections are estimated with respect to the above mass corrections. Both the $K^{-} \pi^{+} \pi^{-} \nu_{\tau}$ and the $K^{-} K^{+} \pi^{-} \nu_{\tau}$ modes have similar uncertainties: $5.4 \%$ and $4.1 \%$. For the $K^{-} \pi^{+} \pi^{-} \pi^{0} \nu_{\tau}$ and the $K^{-} K^{+} \pi^{-} \pi^{0} \nu_{\tau}$ modes, low statistics forbid any investigation of the decay dynamics. A conservative uncertainty of $10 \%$ is assigned for both modes.

\section{Investigation of mass spectra}

The mass spectra for the $K^{-} \pi^{+} \pi^{-}$and the $K^{+} K^{-} \pi^{-}$decay modes are investigated. Signals for both channels are isolated by utilizing the kaon identification estimator defined in (5) and with kinematic cuts. Feedthrough backgrounds are simulated with a statistics four times larger than the data. Because of statistics, the investigation of mass spectra can only be done for the channels with no $\pi^{0}$.

\subsection{Resonances in $\tau^{-} \rightarrow K^{-} K^{+} \pi^{-} \nu_{\tau}$}

The kaon momentum spectrum is quite hard in three-prong $\tau$ decay (Fig. 7). To tag the same-sign pion, tracks with momenta below $7 \mathrm{GeV} / c$ or $P_{\pi} \geq 0.1$ are classified as pions. The kaon momenta must be greater than $7 \mathrm{GeV} / c$ and the kaon probabilities for the samesign and the opposite-sign tracks should be more than 0.5 and 0.9 , respectively. Sixty-nine candidates survive all these requirements with about $25 \%$ background, mainly from the $\pi^{-} \pi^{+} \pi^{-}$decay mode. Fig. 11 shows the invariant mass spectra of $K^{+} \pi^{-}, K^{+} K^{-}$and $K^{-} K^{+} \pi^{-}$systems in the $K^{-} K^{+} \pi^{-} \nu_{\tau}$ data sample. The Monte Carlo prediction in Ref [4] and the estimated feedthrough background are also shown. A clear $K^{*}$ signal is present. A $K^{*}$ Breit-Wigner function together with a linear term describing the background are used to fit the $K \pi$ mass spectrum, giving $(87 \pm 13) \%$ for the $K^{*} K^{-}$component. A systematic mass shift of $(+70 \pm 20) \mathrm{MeV} / c^{2}$ is found in the $K^{-} K^{+} \pi^{-}$invariant mass spectrum, compared to the prediction, and results in a correction to the model when computing the efficiencies listed in Table 1. 

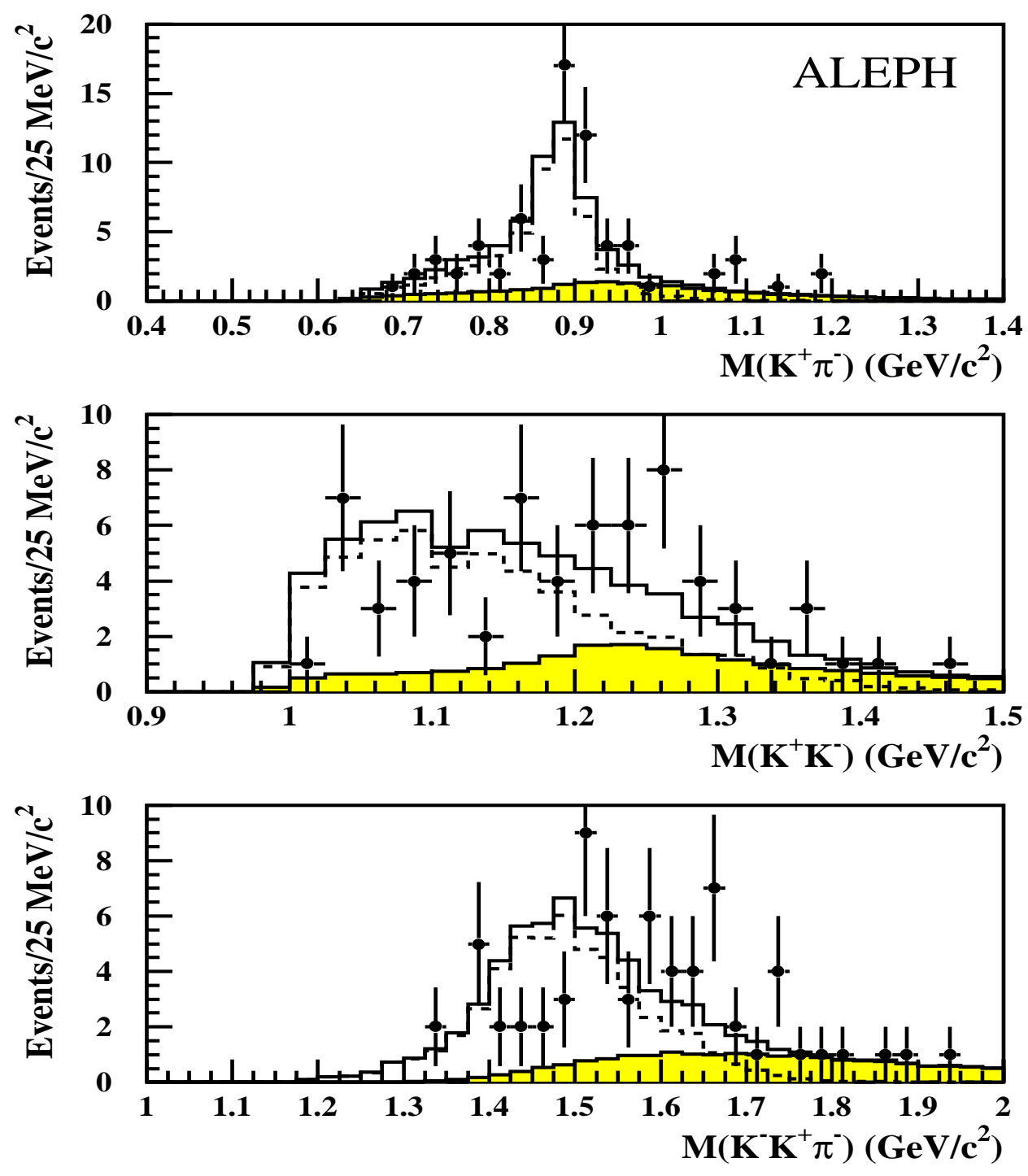

Figure 11: The invariant mass spectra for the $\tau^{-} \rightarrow K^{-} K^{+} \pi^{-} \nu_{\tau}$ sample in data (dots with error bars) and Monte Carlo simulation (histogram). The $K^{-} K^{+} \pi^{-}$signal predicted by the model in Ref [4] is shown in the dashed histogram and the $\tau$ feedthrough background is given by the shaded histogram including $\pi / K$ misidentification. 


\subsection{Resonances in $\tau^{-} \rightarrow K^{-} \pi^{+} \pi^{-} \nu_{\tau}$}

The resonance structures existing in the $K^{-} \pi^{+} \pi^{-} \nu_{\tau}$ decay mode have also been investigated. A $K^{-} \pi^{+} \pi^{-} \nu_{\tau}$ candidate is required to have a same-sign kaon with $p \geq$ $7 \mathrm{GeV} / c$ and $P_{K} \geq 0.95$, and one same-sign pion with either $p \leq 7 \mathrm{GeV} / c$ or satisfying $P_{\pi} \geq 0.05$ and the other opposite-sign pion satisfying $P_{\pi} \geq 0.8$. There are 148 candidates passing all the above cuts with about $50 \%$ expected background. The Monte Carlo shows that the majority of background events are from the $3 \pi^{-} \nu_{\tau}$ and the $K^{0} K^{-} \nu_{\tau}$ decay modes.

In Fig. 12 a clear $K^{*}$ signal is seen. Theoretically, this $K^{*} \pi$ channel is thought to proceed through an axial vector current via the $K_{1}(1270)$ and $K_{1}(1400)$ resonances. The $K^{-} \pi^{+} \pi^{-}$invariant mass distribution does not support a $K_{1}(1400)$ dominance. Instead, a broad structure is observed with a mean value around $1.3 \mathrm{GeV} / c^{2}$, indicating another contribution besides $K_{1}(1400)$. The $K_{1}(1270)$, however, decays to $\rho K$ with a branching ratio of $(42 \pm 6) \%$ [12]. In the invariant mass spectrum of the $\pi^{+} \pi^{-}$system, the $K^{*} \pi$ reflection is found to peak up at $0.5 \mathrm{GeV} / c^{2}$ and decreases quickly with mass. Subtracting all the background gives some excess around the $\rho$ signal region in Fig. 13, in which the shape of $3 \pi$ background is obtained directly from data, since some small discrepancies are observed between the $a_{1}$ simulation and the data in the $\pi \pi$ mass spectrum. An incoherent $\rho$ Breit-Wigner signal and a $K^{*} \pi$ reflection term are used to describe the invariant mass of the two-pion system, giving $(35 \pm 11) \%$ for the $\rho K$ component. This value is indicative of a relatively large $K_{1}(1270)$ component. A more quantitative assessment of this observation needs a further study, which also takes also into account the decay channel $\tau^{-} \rightarrow \bar{K}^{0} \pi^{-} \pi^{0} \nu_{\tau}$.

\section{Results and discussion}

The branching ratios for the five channels studied in three-prong final states with charged kaons are summarized in Table 5 and Fig. 14. The results for $B\left(\tau^{-} \rightarrow K^{-} \pi^{+} \pi^{-} \nu_{\tau}\right)=$ $(2.14 \pm 0.47) \times 10^{-3}$ and $B\left(\tau^{-} \rightarrow K^{-} K^{+} \pi^{-} \nu_{\tau}\right)=(1.63 \pm 0.27) \times 10^{-3}$ represent a substantial improvement in precision over the present experimental situation $[13,14,15$, 16]. Also the channels $\tau^{-} \rightarrow K^{-} \pi^{+} \pi^{-} \pi^{0} \nu_{\tau}$ and $\tau^{-} \rightarrow K^{-} K^{+} \pi^{-} \pi^{0} \nu_{\tau}$ are identified and their branching ratios are measured for the first time. In order to compare the new measurements with existing experimental information, the sums of the branching ratios are given:

$$
\begin{aligned}
& B\left(\tau^{-} \rightarrow K^{-} \pi^{+} \pi^{-} \geq 0 \pi^{0} \nu_{\tau}\right)=(2.75 \pm 0.64) \times 10^{-3} \\
& B\left(\tau^{-} \rightarrow K^{-} K^{+} \pi^{-} \geq 0 \pi^{0} \nu_{\tau}\right)=(2.38 \pm 0.42) \times 10^{-3}
\end{aligned}
$$

to be compared with the corresponding world-average values $\left(3.9_{-1.6}^{+1.9}\right) \times 10^{-3}$ and $\left(1.5_{-0.8}^{+0.9}\right) \times 10^{-3}[12]$.

The measured value for $B\left(\tau^{-} \rightarrow K^{-} \pi^{+} \pi^{-} \nu_{\tau}\right)$ is three times smaller than the prediction of $7.7 \times 10^{-3}$ by Finkemeier and Mirkes [5], but is consistent with the prediction of $1.8 \times 10^{-3}$ by $\mathrm{Li}$ [6]. The result for $B\left(\tau^{-} \rightarrow K^{-} K^{+} \pi^{-} \nu_{\tau}\right)$ is in qualitative agreement with the predictions of $2.0 \times 10^{-3}$ by Finkemeier and Mirkes [5] and $2.6 \times 10^{-3}$ by Li [6]. 

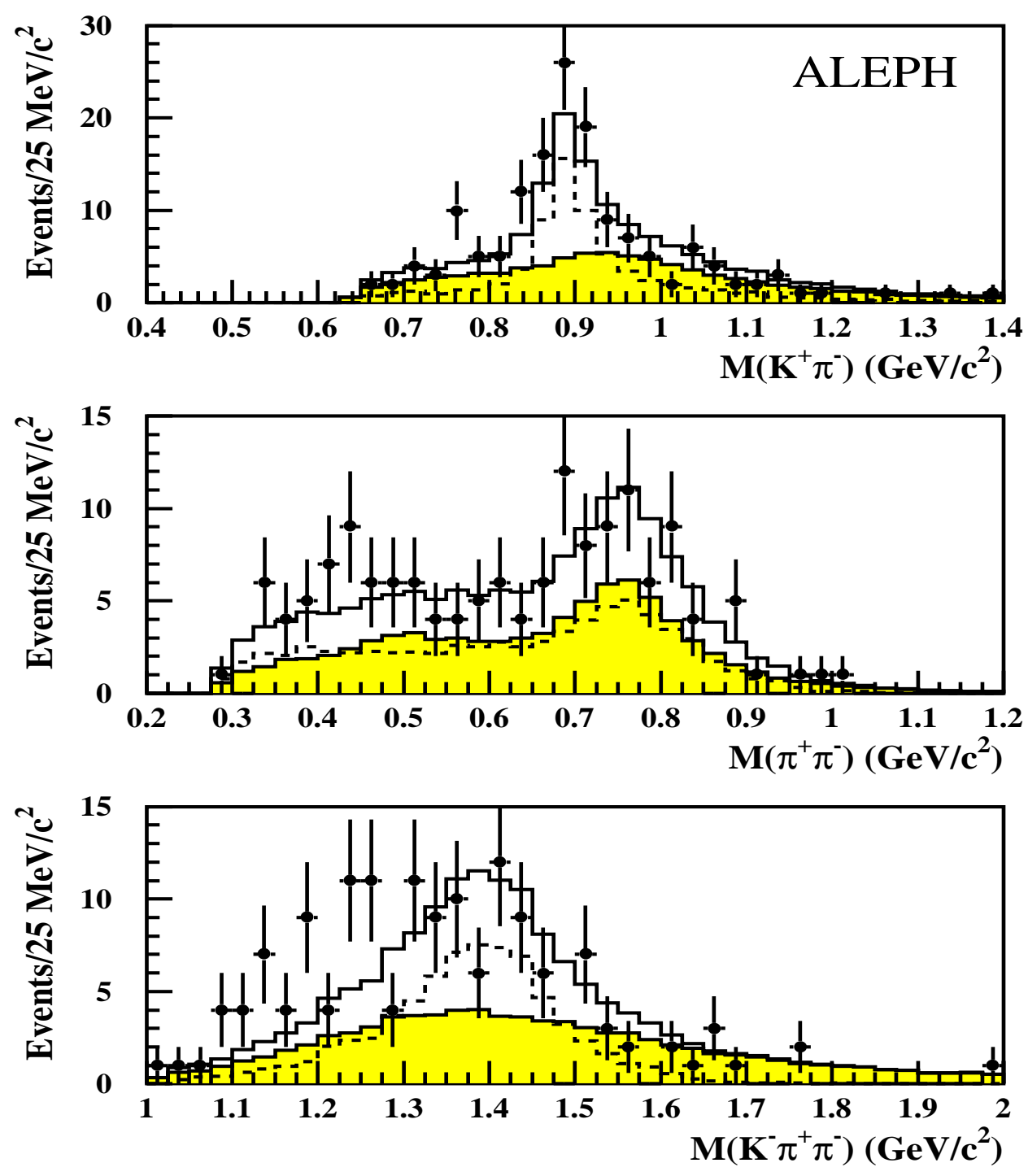

Figure 12: The invariant mass spectra for the $\tau^{-} \rightarrow K^{-} \pi^{+} \pi^{-} \nu_{\tau}$ sample in data (dots with error bars) and Monte Carlo (histogram). The $K^{-} \pi^{+} \pi^{-}$signal predicted by the model of Ref [4] is shown in the dashed histogram and the expected $\tau$ feedthrough background is given by the shaded histogram. 


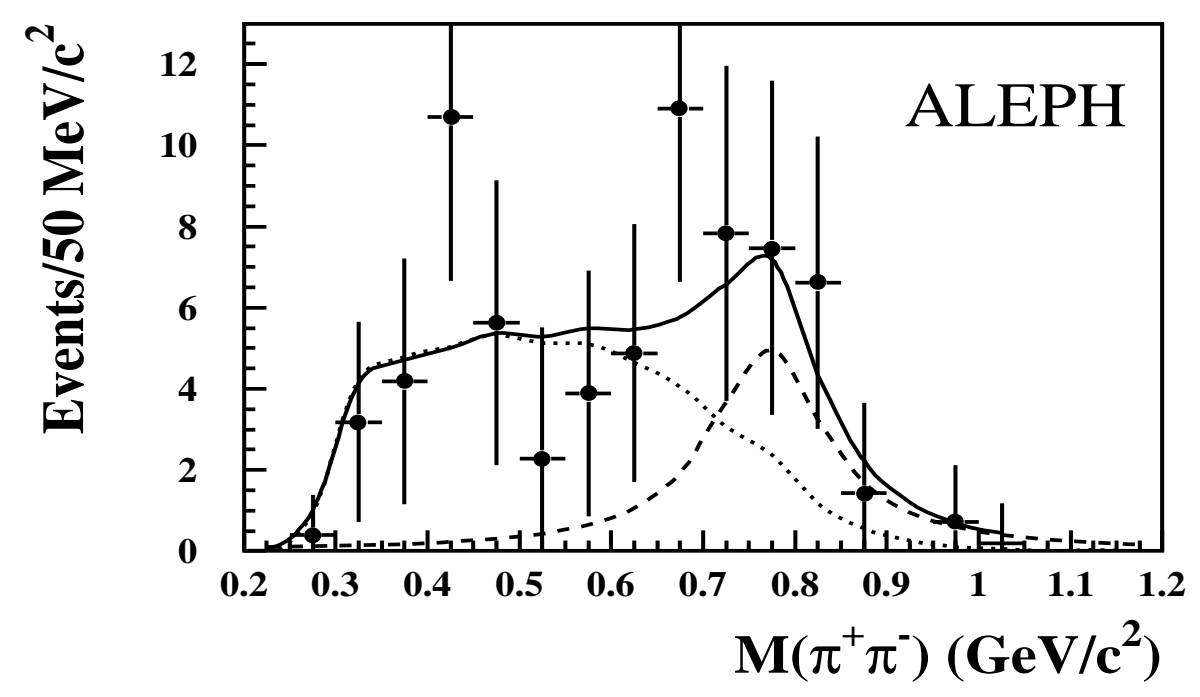

Figure 13: The $\pi^{+} \pi^{-}$invariant mass after background subtraction in the decay $\tau^{-} \rightarrow K^{-} \pi^{+} \pi^{-} \nu_{\tau}$. The fit function is the sum of a $\rho$ Breit-Wigner form and the expected $K^{*} \pi$ reflection.

It is possible to compare the partial rates involving $K^{*}$ production found in this analysis (Fig. 14)

$$
\begin{aligned}
& B\left(\tau^{-} \rightarrow \bar{K}^{* 0} \pi^{-} \nu_{\tau}\right)=(2.09 \pm 0.58) \times 10^{-3}, \\
& B\left(\tau^{-} \rightarrow K^{* 0} K^{-} \nu_{\tau}\right)=(2.13 \pm 0.48) \times 10^{-3},
\end{aligned}
$$

with the earlier measurements relying on a fit of the $K^{*}$ resonance over a phenomenological background: $B\left(\tau^{-} \rightarrow \bar{K}^{* 0} \pi^{-} \nu_{\tau}\right)=(2.5 \pm 1.1) \times 10^{-3}$ and $B\left(\tau^{-} \rightarrow K^{* 0} K^{-} \nu_{\tau}\right)=$ $(2.0 \pm 0.6) \times 10^{-3}$ by ARGUS [16], and $B\left(\tau^{-} \rightarrow \bar{K}^{* 0} \pi^{-} \nu_{\tau}\right)=(3.8 \pm 1.7) \times 10^{-3}$ and $B\left(\tau^{-} \rightarrow K^{* 0} K^{-} \nu_{\tau}\right)=(3.2 \pm 1.4) \times 10^{-3}$ by CLEO [14]. A good agreement is observed.

The $95 \%$ C.L. upper limit of $0.19 \times 10^{-3}$ obtained on the $\tau^{-} \rightarrow K^{-} K^{+} K^{-} \nu_{\tau}$ branching ratio is considerably smaller than the previous limit of $2.1 \times 10^{-3}$ from TPC [15]. The theoretical estimate is of the order $10^{-6}[4]$.

The physics implication of the present results will be discussed in a subsequent paper taking into account the ALEPH measurements of isospin-related final states $\bar{K}^{0} \pi^{-} \pi^{0} \nu_{\tau}$, $K^{-} \pi^{0} \pi^{0} \nu_{\tau}, \bar{K}^{0} K^{0} \pi^{-} \nu_{\tau}$ and $K^{0} K^{-} \pi^{0} \nu_{\tau}$.

\section{Conclusion}

Branching ratios for the decays of $\tau$ leptons into three charged particles including kaons are obtained, separating final states with an additional $\pi^{0}$. The results, listed in Table 5 and shown in Fig. 14, are more precise than the current world averages and include for the first time channels with up to four hadrons in the final states involving kaons. 

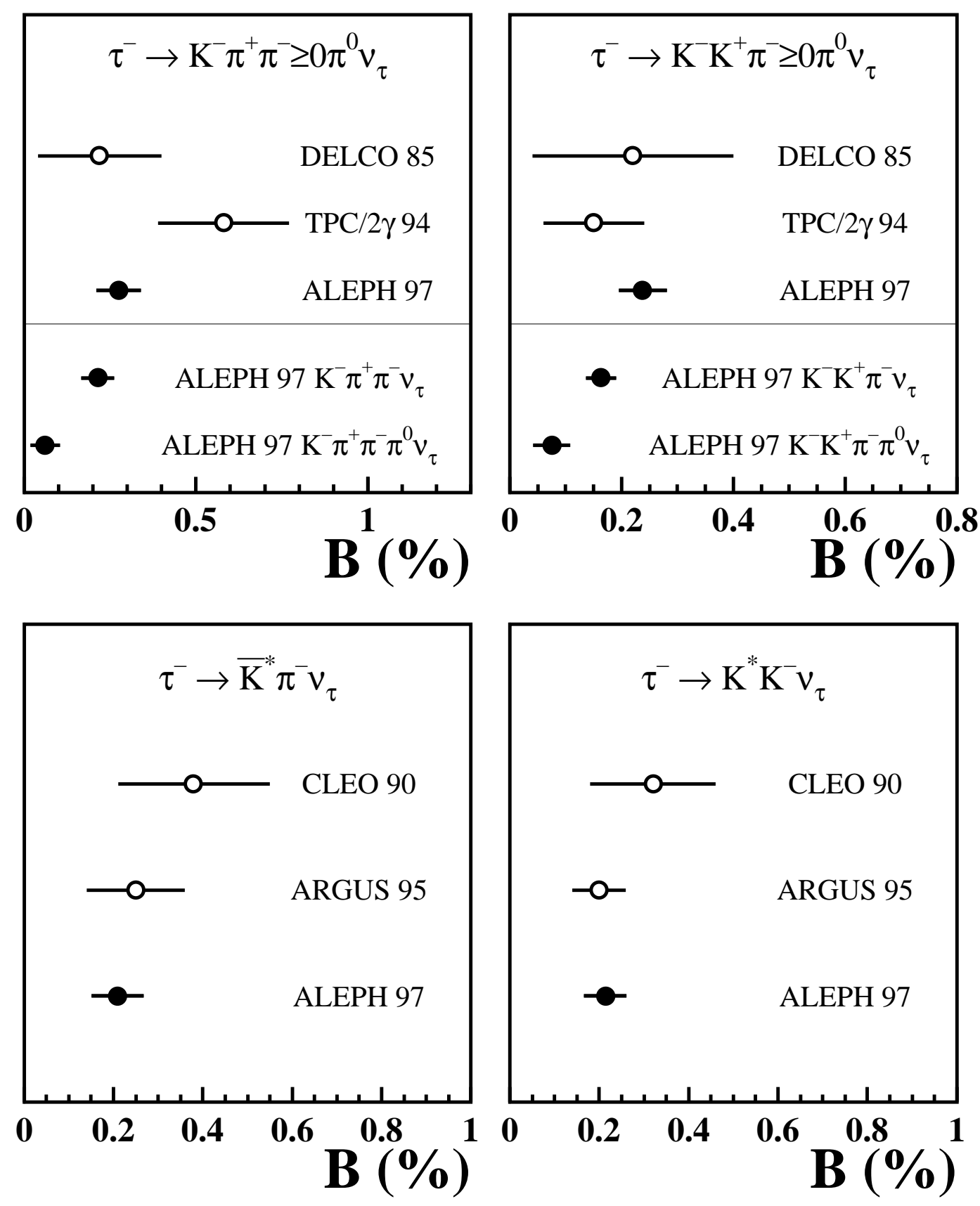

Figure 14: Comparison of the results of the present analysis (black dots) with the published branching ratios for $\tau^{-} \rightarrow K^{-} \pi^{+} \pi^{-} \geq 0 \pi^{0} \nu_{\tau}, \tau^{-} \rightarrow K^{-} K^{+} \pi^{-} \geq 0 \pi^{0} \nu_{\tau}, \tau^{-} \rightarrow \bar{K}^{* 0} \pi^{-} \nu_{\tau}$ and $\tau^{-} \rightarrow K^{* 0} K^{-} \nu_{\tau}[13,14,15,16]$. 


\begin{tabular}{|l|c|}
\hline \hline Mode & $\mathrm{B}\left(\times 10^{-3}\right)$ \\
\hline$K^{-} \pi^{+} \pi^{-} \nu_{\tau}$ & $2.14 \pm 0.37 \pm 0.29$ \\
\hline$K^{-} K^{+} \pi^{-} \nu_{\tau}$ & $1.63 \pm 0.21 \pm 0.17$ \\
\hline$K^{-} K^{+} K^{-} \nu_{\tau}$ & $<0.19$ (95\% C.L.) \\
\hline$K^{-} \pi^{+} \pi^{-} \pi^{0} \nu_{\tau}$ & $0.61 \pm 0.39 \pm 0.18$ \\
\hline$K^{-} K^{+} \pi^{-} \pi^{0} \nu_{\tau}$ & $0.75 \pm 0.29 \pm 0.15$ \\
\hline \hline
\end{tabular}

Table 5: Summary of branching ratios.

\section{Acknowledgements}

We wish to thank our colleagues from the accelerator divisions for the successful operation of the LEP machine, and the engineers and technical staff in all our institutions for their contribution to the good performance of ALEPH. Those of us from non-member states thank CERN for its hospitality.

\section{References}

[1] E. Braaten, S. Narison, and A. Pich, Nucl. Phys. B373 (1992) 581;

S. Narison and A. Pich, Phys. Lett. B304 (1993)359;

M. Suzuki, Phys. Rev. D49 (1994) 2634.

[2] M. Suzuki, Phys. Rev. D47 (1993) 1252.

[3] J.H. Kühn and E. Mirkes, Phys. Lett. B286 (1992) 381, Z. Phys. C56 (1992) 661.

[4] R. Decker, E. Mirkes, R. Sauer, and Z. Was, Z. Phys. C58 (1993) 445.

[5] M. Finkemeier and E. Mirkes, Z. Phys. C69 (1996) 243.

[6] B.A. Li, Phys. Rev, D55 (1997) 1436.

[7] ALEPH Collaboration, Nucl. Instr. Methods A360 (1995) 481.

[8] ALEPH Collaboration, Z. Phys. C70 (1996)579.

[9] ALEPH Collaboration, Phys. Lett. B332 (1994)209.

[10] S. Jadach, B.F.L. Ward, and Z. Was, Comp. Phys. Comm. 79 (1994) 503.

S. Jadach et al., Comp. Phys. Comm. 6 (1993) 361.

[11] T. Sjöstrand, Comp. Phys. Comm. 82 (1994) 74.

[12] R.M. Barnett et al., Particle Data Group, Phys. Rev, D54 (1996) 1.

[13] G.B. Mills et al., DELCO Collaboration, Phys. Rev. Lett. 54 (1985) 624. 
[14] M. Goldberg et al., CLEO Collaboration, Phys. Lett. B251 (1990) 223.

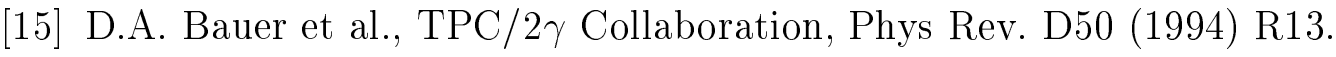

[16] H. Albrecht et al., ARGUS Collaboration, Z. Phys. C68 (1995) 215. 